\title{
The Role of Barium(II) in the Determination of the Absolute Configuration of Chiral Amines by ${ }^{1} \mathrm{H}-\mathrm{NMR}$
}

Rosa García, ${ }^{\dagger}$ José M. Seco, ${ }^{\dagger}$ Saulo A. Vázquez, ${ }^{\dagger}$ Emilio Quiñoá ${ }^{\dagger}$ and Ricardo Riguera ${ }^{\dagger *}$

†Departamento de Química Orgánica and *Departamento de Química Física, Facultad de Química and ${ }^{\dagger}$ Unidad de RMN de Biomoléculas Asociada al CSIC, Universidad de

Santiago de Compostela,

E-15782 Santiago de Compostela, Spain.

ricardo@usc.es

\section{Supporting Information Available}

\section{Table of Contents}

- Experimental Section (Computational Methods, General Procedures, General

Procedure for Configuration Assignment, NMR Spectroscopy, Spectroscopic Data of Compounds $(S)-\mathbf{4},(R)$ and $(S)-\mathbf{5},(R)$ and $(S)-\mathbf{1 2},(R)$ and $(S)-\mathbf{1 3},(R)$ and $(S)-\mathbf{1 4},(R)$ and $(S)-\mathbf{1 8},(R)$ and $(S)-\mathbf{1 9},(R)$ and $(S)-20,(R)$ and $(S)-21,(R)$ and $(S)-22,(R)$ and $(S)-24)$

Page S2

— Figure 1-SI

Page S12

— Figure 2-SI

Page S13

— Geometries and total energies calculated in this work with Gaussian98

Page S14 


\section{Experimental Section}

Computational methods. Density functional theory (DFT) calculations were performed to study the conformational preferences of the $(R)$-MPA amide of propan-2-amine (used as a model compound) as well as its complexes with $\mathrm{Ba}^{2+}$. In particular, we employed the B3LYP method, which combines Becke's three-parameter nonlocal hybrid exchange potential ${ }^{[26]}$ with the nonlocal correlation functional of Lee, Yang, and Parr. ${ }^{27}$ This formalism has been found to be very reliable as far as the description of ion-molecule complexes is concerned. ${ }^{28}$ The basis set employed in the calculations was the standard LanL2DZ, which consists on the Dunning/Huzinaga full double zeta (D95) ${ }^{29}$ for first row, and Los Alamos effective core potentials plus double zeta functions on $\mathrm{Na}-\mathrm{Bi}^{30-32}$

For the conformers of the (R)-MPA amide of propan-2-amine, solvent effects were considered using the Onsager model, ${ }^{20,33}$ which considers a polarizable reference molecule as being in a vacuum spherical cavity. The surroundings of the molecule are treated as a continuum with the macroscopic dielectric constant of the solvent $(\varepsilon=36.64$ for acetonitrile at room temperature). The reference molecule is treated as a point dipole placed in the center of the cavity. A dipole in the molecule will induce a dipole in the medium, and the electric field applied by the solvent dipole will in turn interact with the molecular dipole, leading to net stabilization. With this model, full geometry optimizations were performed by using the self-consistent reaction field method. ${ }^{33}$ Attempts were made to calculate the conformational energies for the complexes of $\mathrm{Ba}^{2+}$ 
by using the Onsager solvation model, but in general success was not achieved. All the calculations were performed with the Gaussian98 series of programs. ${ }^{34}$

General Procedures. The MPA amides were prepared by treatment of the amine (1.0 equivalent) with the corresponding $(R)$ and $(S)$-MPA (1.0 equivalents) in the presence of 1-(3-dimethylaminopropyl)-3-ethylcarbodiimide hydrochloride (EDC, 1.2 equivalents) and DMAP (1.0 equivalents) in dry $\mathrm{CH}_{2} \mathrm{Cl}_{2}$, and under a nitrogen atmosphere. The reaction was stirred at r.t. for $2 \mathrm{~h}$. The organic layer was then washed sequentially with water, $\mathrm{HCl}(1 \mathrm{M})$, water, $\mathrm{NaHCO}_{3}$ (sat) and water. Next, it was dried with $\mathrm{Na}_{2} \mathrm{SO}_{4}$ (anh.) and concentrated under reduced pressure to yield the MPA amide. Final purification was achieved by HPLC (m-Porasil, $3 \mathrm{~mm}$ x $250 \mathrm{~mm}$ or Spherisorb S5W 5mm, hexaneethyl acetate). All the compounds were characterized by optical rotation, NMR (1D, 2D) and $\mathrm{MS}(\mathrm{EI})$.

For experimental details on compounds: 9, 11, 15 and 17see Ref. 5a.

General Procedure for Configuration Assignment. The amine is reacted with the either the $(R)$ or the $(S)$-MPA reagent, the spectrum of the resulting MPA amide is recorded in $\mathrm{MeCN}^{-\mathrm{d}_{3}}$ (i.e. $5 \mathrm{mg}$ of amide in $0.5 \mathrm{~mL}$ of $\mathrm{MeCN}-\mathrm{d}_{3}$ ), and the signals of $\mathrm{L}_{1}$ and $\mathrm{L}_{2}$ assigned. Solid anhydrous $\mathrm{Ba}\left(\mathrm{ClO}_{4}\right)_{2}$ is added to the NMR tube until saturation is attained (about $200 \mathrm{mg}$ per tube) and a new spectrum recorded. The signals are assigned and the $\Delta \delta^{\mathrm{Ba}}$ values and signs are calculated. The models shown in Figure 15 are used to place $\mathrm{L}_{1}$ and $\mathrm{L}_{2}$ in space in accordance with the signs of $\Delta \delta$. $^{\mathrm{Ba}}$ 
NMR spectroscopy. ${ }^{1} \mathrm{H}$ and ${ }^{13} \mathrm{C}$ NMR spectra of samples in $\mathrm{CDCl}_{3}$ were recorded in 500 and $250 \mathrm{MHz}$. Chemical shifts ( ppm) are internally referenced to the TMS signal $(0 \mathrm{ppm})$ in all cases. $J$ values are recorded in $\mathrm{Hz}$.

1D ${ }^{1} \mathrm{H}$ NMR spectra. Size $32 \mathrm{~K}$, pulse length $2.8 \mu \mathrm{s}\left(30^{\circ}\right), 16$ acquisitions.

$1 \mathrm{D}{ }^{13} \mathrm{C}$ NMR spectra. Size $64 \mathrm{~K}$, pulse length $3.5 \mu \mathrm{s}\left(30^{\circ}\right), 1024$ acquisitions.

2D COSY Spectra. Sequence: D1-90-t1-90-t2; relaxation delay D1 $=0.5 \mathrm{~s} ; 90^{\circ}$ pulse $8.5 \mu \mathrm{s}$.

2D NOESY Spectra. Sequence: D1-90-t1-90- $\tau_{\text {mix }}-90-\mathrm{t} 2$; relaxation delay D1 $=0.5 \mathrm{~s}$; mixing time $\left(\tau_{\text {mix }}\right) 0.5 \mathrm{~s}, 90^{\circ}$ pulse $8.5 \mu \mathrm{s}$; TPPI-mode, $\mathrm{NS}=64$. 
(-)-Isopinocampheyl-(S)-2-methoxy-2-phenylacetamide [(S)-4]

$[\alpha]_{\mathrm{D}}=+90.00\left(\mathrm{c}=0.004, \mathrm{CHCl}_{3}\right) ;{ }^{1} \mathrm{H}$ NMR $\left(250.13 \mathrm{MHz}, \mathrm{CDCl}_{3}\right) \delta(\mathrm{ppm}) 0.92(\mathrm{~d}$, $\mathrm{J}=10.04 \mathrm{~Hz}, 1 \mathrm{H}), 1.02(\mathrm{~d}, \mathrm{~J}=7.85 \mathrm{~Hz}, 3 \mathrm{H}), 1.03$ (s, 3H), 1.22 (s, 3H), 1.60 (ddd, J=2.19, 6.28, $13.97 \mathrm{~Hz}, 1 \mathrm{H}), 1.75-1.88(\mathrm{~m}, 2 \mathrm{H}), 1.98(\mathrm{~m}, 1 \mathrm{H}), 2.43(\mathrm{~m}, 1 \mathrm{H}), 2.62$ (dddd, J=2.20, 3.37, 10.20, $13.81 \mathrm{~Hz}, 1 \mathrm{H}), 3.36(\mathrm{~s}, 3 \mathrm{H}), 4.26(\mathrm{~m}, 1 \mathrm{H}), 4.60(\mathrm{~s}, 1 \mathrm{H}), 6.63(\mathrm{br}, \mathrm{J}=8.17$ $\mathrm{Hz}, 1 \mathrm{H}), 7.30-7.40(\mathrm{~m}, 5 \mathrm{H}) ;{ }^{13} \mathrm{C} \mathrm{NMR}\left(62.9 \mathrm{MHz}, \mathrm{CDCl}_{3}\right) \delta(\mathrm{ppm}) 21.1,23.7,28.5$, $35.8,37.4,38.8,42.0,46.7,47.7,48.2,57.5,84.2,127.4,128.8,128.9,137.75,170.0$ MS (EI) $\mathrm{m} / \mathrm{z} \% 301\left(\mathrm{M}^{+}\right)$.

(2S)-N-sec-Butyl-(R)-2-methoxy-2-phenylacetamide [(R)-5]

$[\alpha]_{\mathrm{D}}=-101\left(\mathrm{c}=0.014, \mathrm{CH}_{3} \mathrm{Cl}\right) ;{ }^{1} \mathrm{H} \mathrm{NMR}\left(250.13 \mathrm{MHz}, \mathrm{CH}_{3} \mathrm{CN}-d_{3}\right) \delta(\mathrm{ppm}) 0.78(\mathrm{t}$, $\mathrm{J}=7.41 \mathrm{~Hz}, 3 \mathrm{H}), 1.11$ (d, J=6.64 Hz, 3H), $1.43(\mathrm{~m}, 2 \mathrm{H}), 3.29(\mathrm{~s}, 3 \mathrm{H}), 3.77(\mathrm{~m}, 1 \mathrm{H}), 4.55$ $(\mathrm{s}, 1 \mathrm{H}), 6.88(\mathrm{br}, 1 \mathrm{H}), 7.26-7.45(\mathrm{~m}, 5 \mathrm{H}) ;{ }^{13} \mathrm{C} \mathrm{NMR}\left(62.9 \mathrm{MHz}, \mathrm{CH}_{3} \mathrm{CN}-d_{3}\right) \delta(\mathrm{ppm})$ 9.6, 19.49, 28.8, 45.7, 56.2, 83.4, 126.8, 127.8, 127.9, 137.9, 169.1; MS (EI) m/z \% 220 $\left(\mathrm{M}^{+}\right)$.

(2S)-N-sec-Butyl-(S)-2-methoxy-2-phenylacetamide [(S)-5]

$[\alpha]_{\mathrm{D}}=+77.89\left(\mathrm{c}=0.0093, \mathrm{CH}_{3} \mathrm{Cl}\right) ;{ }^{1} \mathrm{H} \mathrm{NMR}\left(250.13 \mathrm{MHz}, \mathrm{CH}_{3} \mathrm{CN}-d_{3}\right) \delta(\mathrm{ppm}) 0.87(\mathrm{t}$, $\mathrm{J}=7.42 \mathrm{~Hz}, 3 \mathrm{H}), 1.05(\mathrm{~d}, \mathrm{~J}=6.64 \mathrm{~Hz}, 3 \mathrm{H}), 1.48(\mathrm{~m}, 2 \mathrm{H}), 3.31(\mathrm{~s}, 3 \mathrm{H}), 3.76(\mathrm{~m}, 1 \mathrm{H}), 4.56$ $(\mathrm{s}, 1 \mathrm{H}), 6.88(\mathrm{br}, 1 \mathrm{H}), 7.21-7.46(\mathrm{~m}, 5 \mathrm{H}) ;{ }^{13} \mathrm{C} \mathrm{NMR}\left(62.9 \mathrm{MHz}, \mathrm{CH}_{3} \mathrm{CN}-d_{3}\right) \delta(\mathrm{ppm})$ $9.7,19.3,28.7,45.8,56.3,83.3,126.8,127.8,128.0,137.9,169.2 ; \mathrm{MS}(\mathrm{EI}) \mathrm{m} / \mathrm{z} \% 220$ $\left(\mathrm{M}^{+}\right)$.

$(R)-(1-C y c l o h e x y l e t h y l)-(R)-2-m e t h o x y-2-$ phenylacetamide $[(R)-12]$

$[\alpha]_{\mathrm{D}}=-49.33(\mathrm{c}=0.003, \mathrm{EtOH}) ;{ }^{1} \mathrm{H}$ NMR $\left(250.13 \mathrm{MHz}, \mathrm{CH}_{3} \mathrm{CN}-d_{3}\right) \delta(\mathrm{ppm})$ 0.89-1.09 $(\mathrm{m}, 2 \mathrm{H}), 1.03(\mathrm{~d}, \mathrm{~J}=6.60 \mathrm{~Hz}, 3 \mathrm{H}), 1.12-1.45(\mathrm{~m}, 4 \mathrm{H}), 1.58-1.81(\mathrm{~m}, 5 \mathrm{H}), 3.33(\mathrm{~s}, 3 \mathrm{H})$ 
$3.68(\mathrm{~s}, 1 \mathrm{H}), 4.56(\mathrm{~s}, 1 \mathrm{H}), 6.86(\mathrm{br}, 1 \mathrm{H}), 7.25-7.43(\mathrm{~m}, 5 \mathrm{H}) ;{ }^{13} \mathrm{C}$ NMR $(62.9 \mathrm{MHz}$, $\left.\mathrm{CDCl}_{3}\right) \delta(\mathrm{ppm}) 18.3,26.6,26.8,29.4,29.5,43.4,49.3,57.7,84.2,127.4,128.7,128.9$, 137.7, 170.1; MS (EI) m/z \% $275\left(\mathrm{M}^{+}\right)$.

$(R)-(1-C y c l o h e x y l e t h y l)-(S)-2-m e t h o x y-2-p h e n y l a c e t a m i d e ~[(S)-12]$

$[\alpha]_{\mathrm{D}}=+62.66(\mathrm{c}=0.006, \mathrm{EtOH}) ;{ }^{1} \mathrm{H} \mathrm{NMR}\left(250.13 \mathrm{MHz}, \mathrm{CH}_{3} \mathrm{CN}-d_{3}\right) \delta(\mathrm{ppm}) 0.75-$ $0.98(\mathrm{~m}, 2 \mathrm{H}), 1.09(\mathrm{~d}, \mathrm{~J}=6.60 \mathrm{~Hz}, 3 \mathrm{H}), 1.06-1.38(\mathrm{~m}, 4 \mathrm{H}), 1.53-1.74(\mathrm{~m}, 5 \mathrm{H}), 3.31(\mathrm{~s}$, $3 \mathrm{H}), 3.68(\mathrm{~s}, 1 \mathrm{H}), 4.54(\mathrm{~s}, 1 \mathrm{H}), 6.84(\mathrm{br}, 1 \mathrm{H}), 7.30-7.43(\mathrm{~m}, 5 \mathrm{H}) ;{ }^{13} \mathrm{C}$ NMR $(62.9$ $\left.\mathrm{MHz}, \mathrm{CDCl}_{3}\right) \delta 18.0,26.1,26.3,28.7,29.1,43.1,48.8,57.1,84.0,127.0,128.3,128.5$, 137.3, 169.0; MS (EI) m/z \% $275\left(\mathrm{M}^{+}\right)$.

$(S)-(+)-I n d a n-1-y l-(R)-2-m e t h o x y-2-p h e n y l a c e t a m i d e ~[(R)-13]$

$[\alpha]_{\mathrm{D}}=-142.57\left(\mathrm{c}=0.014, \mathrm{CHCl}_{3}\right) ;{ }^{1} \mathrm{H} \mathrm{NMR}\left(250.13 \mathrm{MHz}, \mathrm{CH}_{3} \mathrm{CN}-d_{3}\right) \delta(\mathrm{ppm}) 1.90$ (m, 1H), $2.48(\mathrm{~m}, 1 \mathrm{H}), 2.78-3.05(\mathrm{~m}, 2 \mathrm{H}), 3.32(\mathrm{~s}, 3 \mathrm{H}), 4.65(\mathrm{~s}, 1 \mathrm{H}), 5.37(\mathrm{q}, \mathrm{J}=8.10 \mathrm{~Hz}$, 1H), $7.02(\mathrm{~d}, \mathrm{~J}=6.91 \mathrm{~Hz}, 1 \mathrm{H}), 7.07-7.46(\mathrm{~m}, 9 \mathrm{H}) ;{ }^{13} \mathrm{C} \mathrm{NMR}\left(62.9 \mathrm{MHz}, \mathrm{CDCl}_{3}\right) \delta 30.7$, $34.5,54.3,57.5,74.8,84.2,124.3,125.2,127.2,127.5,128.3,128.9,129.0,137.6$, 143.5, 143.7, 170.8; MS (EI) m/z \% $281\left(\mathrm{M}^{+}\right)$.

$(S)-(+)-I n d a n-1-y l-(S)-2-m e t h o x y-2-$ phenylacetamide $[(S)-13]$

$[\alpha]_{\mathrm{D}}=+34.25\left(\mathrm{c}=0.008, \mathrm{CHCl}_{3}\right) ;{ }^{1} \mathrm{H} \mathrm{NMR}\left(250.13 \mathrm{MHz}, \mathrm{CH}_{3} \mathrm{CN}-d_{3}\right) \delta(\mathrm{ppm}) 1.85$ $(\mathrm{m}, 1 \mathrm{H}), 2.42(\mathrm{~m}, 1 \mathrm{H}), 2.77-3.05(\mathrm{~m}, 2 \mathrm{H}), 3.33(\mathrm{~s}, 3 \mathrm{H}), 4.67(\mathrm{~s}, 1 \mathrm{H}), 5.37(\mathrm{q}, \mathrm{J}=8.06$, 1H), 7.16-7.44 (m, 10H); ${ }^{13} \mathrm{C}$ NMR (62.9 MHz, $\left.\mathrm{CDCl}_{3}\right) \delta(\mathrm{ppm}) \quad 30.2,33.9,54.1,57.2$, $74.3,83.8,124.1,124.8,126.8,127.0,128.0,128.5,128.6,137.1,143 ., 143.1,170.3$; MS (EI) $\mathrm{m} / \mathrm{z} \% 281\left(\mathrm{M}^{+}\right)$. 
$[(1 S, 2 R)-1-H y d r o x y-1-p h e n y l p r o p a n-2-y 1]-(R)-2-m e t h o x y-2-p h e n y l a c e t a m i d e ~[(R)-$ 14]

$[\alpha]_{\mathrm{D}}=-13.11\left(\mathrm{c}=0.004, \mathrm{CHCl}_{3}\right) ;{ }^{1} \mathrm{H} \mathrm{NMR}\left(250.13 \mathrm{MHz}, \mathrm{CH}_{3} \mathrm{CN}-d_{3}\right) \delta(\mathrm{ppm}) 0.98(\mathrm{~d}$, $\mathrm{J}=6.59 \mathrm{~Hz}, 3 \mathrm{H}), 3.27(\mathrm{~s}, 3 \mathrm{H}), 3.81(\mathrm{~d}, \mathrm{~J}=4.70 \mathrm{~Hz}, 1 \mathrm{H}), 4.00-4.14(\mathrm{~m}, 1 \mathrm{H}), 4.75(\mathrm{t}$, $\mathrm{J}=4.55 \mathrm{~Hz}, 1 \mathrm{H}), 7.09(\mathrm{br}, 1 \mathrm{H}), 7.24-7.38(\mathrm{~m}, 10 \mathrm{H}) ;{ }^{13} \mathrm{C} \mathrm{NMR}\left(62.9 \mathrm{MHz}, \mathrm{CDCl}_{3}\right)$ $\delta(\mathrm{ppm}) 15.4,50.9,57.1,83.6,126.5,127.0,127.4,128.0,128.4,136.0,140.2,171.1$;

MS (EI) m/z \% $299\left(\mathrm{M}^{+}\right)$.

[(1S,2R)-1-Hydroxy-1-phenylpropan-2-yl]-(S)-2-methoxy-2-phenylacetamide [(S)-14] $[\alpha]_{\mathrm{D}}=+119.25\left(\mathrm{c}=0.014, \mathrm{CHCl}_{3}\right) ;{ }^{1} \mathrm{H} \mathrm{NMR}\left(250.13 \mathrm{MHz}, \mathrm{CH}_{3} \mathrm{CN}-d_{3}\right) \delta(\mathrm{ppm}) 1.07$ $(\mathrm{d}, \mathrm{J}=6.59 \mathrm{~Hz}, 3 \mathrm{H}), 3.26(\mathrm{~s}, 3 \mathrm{H}), 3.85$ (br., 1H), $4.10(\mathrm{~m}, 1 \mathrm{H}), 4.52(\mathrm{~s}, 1 \mathrm{H}), 4.66$ (d, $\mathrm{J}=4.71 \mathrm{~Hz}, 1 \mathrm{H}), 7.09$ (br, 1H), 7.14-7.22 (m, 2H), 7.26-7.36 (m, 8H); ${ }^{13} \mathrm{C}$ NMR $(62.9$

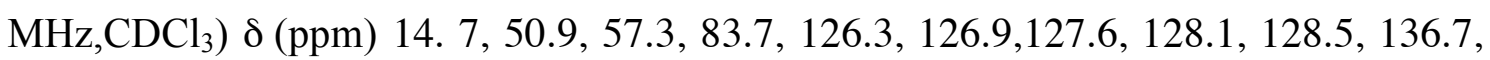
140.5, 171.0; MS (EI) m/z \% $299\left(\mathrm{M}^{+}\right)$.

(R)-Methyl 3-[(S)-2-methoxy-2-phenylacetamido]dodecanoate $[(S)-18]$

$[\alpha]_{\mathrm{D}}=30.20\left(\mathrm{c}=0.0015, \mathrm{CHCl}_{3}\right) ;{ }^{1} \mathrm{H} \mathrm{NMR}\left(250.13 \mathrm{MHz}, \mathrm{CDCl}_{3}\right) \delta(\mathrm{ppm}) 0.88(\mathrm{t}$, $\mathrm{J}=6.55 \mathrm{~Hz}, 3 \mathrm{H}), 1.18-1.38(\mathrm{~m}, 14 \mathrm{H}), 1.44-1.59(\mathrm{~m}, 2 \mathrm{H}), 2.46(\mathrm{~d}, \mathrm{~J}=6.45 \mathrm{~Hz}, 2 \mathrm{H}), 3.31$ (s, 3H), $3.51(\mathrm{~s}, 3 \mathrm{H}), 4.05-4.19(\mathrm{~m}, 1 \mathrm{H}), 4.55(\mathrm{~s}, 1 \mathrm{H}), 7.10(\mathrm{br}, \mathrm{J}=8.10 \mathrm{~Hz}, 1 \mathrm{H}), 7.25-$ $7.35(\mathrm{~m}, 5 \mathrm{H}) ;{ }^{13} \mathrm{C}$ NMR $\left(62.9 \mathrm{MHz}, \mathrm{CDCl}_{3}\right) \delta(\mathrm{ppm})$ 14.1, 22.6, 26.0, 29.2, 29.4, 29.4, $31.8,34.2,38.7,45.6,51.7,57.3,83.9,126.8,128.3,128.5,137.3,170.0,172.0 ; \mathrm{MS}$ 
(EI) $\mathrm{m} / \mathrm{z} \% 377\left(\mathrm{M}^{+}\right)$.

(R)-Methyl 3-[(R)-2-methoxy-2-phenylacetamido]dodecanoate [(R)-18]

$[\alpha]_{\mathrm{D}}=+18.82\left(\mathrm{c}=0.0017, \mathrm{CHCl}_{3}\right) ;{ }^{1} \mathrm{H} \mathrm{NMR}\left(250.13 \mathrm{MHz}, \mathrm{CH}_{3} \mathrm{CN}-d_{3}\right) \delta(\mathrm{ppm}) 0.88$ $(\mathrm{t}, \mathrm{J}=6.66 \mathrm{~Hz}, 3 \mathrm{H}), 1.07-1.22(\mathrm{~m}, 7 \mathrm{H}), 1.40-1.53(\mathrm{~m}, 2 \mathrm{H}), 2.49(\mathrm{~d}, \mathrm{~J}=6.40 \mathrm{~Hz}, 2 \mathrm{H}), 3.32$ (s, 3H), $3.60(\mathrm{~s}, 3 \mathrm{H}), 4.05-4.19(\mathrm{~m}, 1 \mathrm{H}), 4.54(\mathrm{~s}, 1 \mathrm{H}), 7.10(\mathrm{~d}, \mathrm{~J}=9.20 \mathrm{~Hz}, 1 \mathrm{H}), 7.31-7.38$ $(\mathrm{m}, 5 \mathrm{H}) ;{ }^{13} \mathrm{C} \mathrm{NMR}\left(62.9 \mathrm{MHz}, \mathrm{CDCl}_{3}\right) \delta(\mathrm{ppm})$ 14.2, 22.8, 26.1, 29.3, 29.4, 29.5, 31.9, $34.3,38.8,45.7,51.7,57.3,83.9,126.8,128.2,128.4,137.3,169.9,173.4 ;$ MS (EI) m/z $\% 377\left(\mathrm{M}^{+}\right)$.

$N-[(1 S, 2 R)-1-$ Trimethylsilyloxy-1-phenylpropan-2-yl]-(R)-2-methoxy-2-

phenylacetamide $[(R)-19]$

$[\alpha]_{\mathrm{D}}=-65.72\left(\mathrm{c}=0.0241, \mathrm{CHCl}_{3}\right) ;{ }^{1} \mathrm{H} \mathrm{NMR}\left(250.13 \mathrm{MHz}, \mathrm{CH}_{3} \mathrm{CN}-d_{3}\right) \delta(\mathrm{ppm}) 0.05(\mathrm{~s}$, 9H), $0.92(\mathrm{~d}, \mathrm{~J}=6.76 \mathrm{~Hz}, 3 \mathrm{H}), 3.26(\mathrm{~s}, 3 \mathrm{H}), 3.98-4.04(\mathrm{~m}, 1 \mathrm{H}), 4.50(\mathrm{~s}, 1 \mathrm{H}), 4.86(\mathrm{~d}$, $\mathrm{J}=4.40 \mathrm{~Hz}, 1 \mathrm{H}), 7.05$ (br, J=7.93 Hz, 1H), 7.24-7.35 (m, 10H); ${ }^{13} \mathrm{C}$ NMR (62.9 MHz, $\left.\mathrm{CDCl}_{3}\right) \delta(\mathrm{ppm}) \quad 13.6,50.3,57.2,83.7,126.1,126.9,127.1,128.0,128.2,128.4$ 137.01, 141.6, 169.5; MS (EI) m/z \% $371\left(\mathrm{M}^{+}\right)$.

$N-[(1 S, 2 R)-1-T r i m e t h y l s i l y l o x y-1-p h e n y l p r o p a n-2-y l]-(S)-2-m e t h o x y-2-$ phenylacetamide $[(S)-19]$

$[\alpha]_{\mathrm{D}}=+43.33\left(\mathrm{c}=0.0168, \mathrm{CHCl}_{3}\right) ;{ }^{1} \mathrm{H} \mathrm{NMR}\left(250.13 \mathrm{MHz}, \mathrm{CH}_{3} \mathrm{CN}-d_{3}\right) \delta(\mathrm{ppm}) 0.01(\mathrm{~s}$, 9H), $1.03(\mathrm{~d}, \mathrm{~J}=6.68 \mathrm{~Hz}, 3 \mathrm{H}), 3.27(\mathrm{~s}, 3 \mathrm{H}), 3.98-4.07(\mathrm{~m}, 1 \mathrm{H}), 4.51$ (s, 1H), 4.74 (d, $\mathrm{J}=5.25 \mathrm{~Hz}, 1 \mathrm{H}), 7.02(\mathrm{br}, \mathrm{J}=7.84 \mathrm{~Hz}, 1 \mathrm{H}), 7.15-7.51(\mathrm{~m}, 10 \mathrm{H}) ;{ }^{13} \mathrm{C} \mathrm{NMR}(62.9 \mathrm{MHz}$, $\left.\mathrm{CDCl}_{3}\right) \delta(\mathrm{ppm}) \quad 12.9,50.7,57.1,83.8,126.0,126.2,127.5,128.1,128.5,128.6$ 137.12, 
141.8, 169.7; MS (EI) m/z \% $371\left(\mathrm{M}^{+}\right)$.

(S)-Dimethyl 2-[(R)-2-methoxy-2-phenylacetamido]pentanedioate [(R)-20]

$[\alpha]_{\mathrm{D}}=-61.67\left(\mathrm{c}=0.0091, \mathrm{CH}_{3} \mathrm{Cl}\right) ;{ }^{1} \mathrm{H} \mathrm{NMR}\left(250.13 \mathrm{MHz}, \mathrm{CH}_{3} \mathrm{CN}-d_{3}\right) \delta(\mathrm{ppm}) 1.98$ (m, 1H), $2.16(\mathrm{~m}, 1 \mathrm{H}), 2.34(\mathrm{dd}, \mathrm{J}=7.16,7.16 \mathrm{~Hz}, 2 \mathrm{H}), 3.33(\mathrm{~s}, 3 \mathrm{H}), 4.42(\mathrm{~m}, 1 \mathrm{H}), 4.63$ (s, 1H), 7.29-7.41 (m, 5H), $7.46(\mathrm{~d}, \mathrm{~J}=6.76 \mathrm{~Hz}, 1 \mathrm{H}) ;{ }^{13} \mathrm{C} \mathrm{NMR}\left(62.9 \mathrm{MHz}, \mathrm{CH}_{3} \mathrm{CN}-d_{3}\right) \delta$ (ppm) 25.9, 29.4, 50.9, 50.9, 51.5, 56.3, 83.0, 127.1, 128.0, 137.3, 170.2, 171.5, 172.65; MS (EI) $\mathrm{m} / \mathrm{z} \% 322\left(\mathrm{M}^{+}\right)$.

$(S)$-Dimethyl 2-[(S)-2-methoxy-2-phenylacetamido]pentanedioate $[(S)$-20]

$[\alpha]_{\mathrm{D}}=+55.73\left(\mathrm{c}=0.0087, \mathrm{CH}_{3} \mathrm{Cl}\right) ;{ }^{1} \mathrm{H} \mathrm{NMR}\left(250.13 \mathrm{MHz}, \mathrm{CH}_{3} \mathrm{CN}-d_{3}\right) \delta(\mathrm{ppm}) 1.95$

(m, 1H), $2.13(\mathrm{~m}, 1 \mathrm{H}), 2.29(\mathrm{dd}, \mathrm{J}=7.08,7.08 \mathrm{~Hz}, 2 \mathrm{H}), 3.36(\mathrm{~s}, 3 \mathrm{H}), 3.58(\mathrm{~s}, 3 \mathrm{H})$, $3.67(\mathrm{~s}, 3 \mathrm{H}), 4.41(\mathrm{~m}, 1 \mathrm{H}), 4.62(\mathrm{~s}, 1 \mathrm{H}), 7.32-7.41(\mathrm{~m}, 5 \mathrm{H}), 7.45(\mathrm{~d}, \mathrm{~J}=6.95 \mathrm{~Hz}, 1 \mathrm{H}) ;{ }^{13} \mathrm{C}$ NMR (62.9 MHz, $\left.\mathrm{CH}_{3} \mathrm{CN}-d_{3}\right) \delta(\mathrm{ppm}) 26.0,26.3,50.7,51.6,83.2,126.8,128.0,128.1$, 137.35, 170.4, 171.6, 172.6; MS (EI) m/z \% $322\left(\mathrm{M}^{+}\right)$.

(S)-Methyl 3-[(R)-2-methoxy-2-phenylacetamido]dodecanoate [(R)-21]

$[\alpha]_{\mathrm{D}}=-30.20\left(\mathrm{c}=0.0025, \mathrm{CHCl}_{3}\right) ;{ }^{1} \mathrm{H} \mathrm{NMR}\left(250.13 \mathrm{MHz}, \mathrm{CDCl}_{3}\right) \delta(\mathrm{ppm}) 0.88(\mathrm{t}$, $\mathrm{J}=6.55 \mathrm{~Hz}, 3 \mathrm{H}), 1.18-1.38(\mathrm{~m}, 14 \mathrm{H}), 1.44-1.59(\mathrm{~m}, 2 \mathrm{H}), 2.46(\mathrm{~d}, \mathrm{~J}=6.45 \mathrm{~Hz}, 2 \mathrm{H}), 3.31$ (s, 3H), $3.51(\mathrm{~s}, 3 \mathrm{H}), 4.05-4.19(\mathrm{~m}, 1 \mathrm{H}), 4.55(\mathrm{~s}, 1 \mathrm{H}), 7.10(\mathrm{br}, \mathrm{J}=8.10 \mathrm{~Hz}, 1 \mathrm{H}), 7.25-$ $7.35(\mathrm{~m}, 5 \mathrm{H}) ;{ }^{13} \mathrm{C}$ NMR $\left(62.9 \mathrm{MHz}, \mathrm{CDCl}_{3}\right) \delta(\mathrm{ppm})$ 14.1, 22.6, 26.0, 29.2, 29.4, 29.4, $31.8,34.2,38.7,45.6,51.6,57.3,83.9,126.8,128.3,128.5,137.3,170.0,172.0 ;$ MS (EI) $\mathrm{m} / \mathrm{z} \% 377\left(\mathrm{M}^{+}\right)$. 
(S)-Methyl 3-[(S)-2-methoxy-2-phenylacetamido]dodecanoate $[(S)-21]$

$[\alpha]_{\mathrm{D}}=-18.82\left(\mathrm{c}=0.0015, \mathrm{CHCl}_{3}\right) ;{ }^{1} \mathrm{H} \mathrm{NMR}\left(250.13 \mathrm{MHz}, \mathrm{CH}_{3} \mathrm{CN}-d_{3}\right) \delta(\mathrm{ppm}) 0.88(\mathrm{t}$, $\mathrm{J}=6.66 \mathrm{~Hz}, 3 \mathrm{H}), 1.07-1.22(\mathrm{~m}, 7 \mathrm{H}), 1.40-1.53(\mathrm{~m}, 2 \mathrm{H}), 2.49(\mathrm{~d}, \mathrm{~J}=6.40 \mathrm{~Hz}, 2 \mathrm{H}), 3.32(\mathrm{~s}$, 3H), $3.60(\mathrm{~s}, 3 \mathrm{H}), 4.05-4.19(\mathrm{~m}, 1 \mathrm{H}), 4.54(\mathrm{~s}, 1 \mathrm{H}), 7.10(\mathrm{~d}, \mathrm{~J}=9.20 \mathrm{~Hz}, 1 \mathrm{H}), 7.31-7.38$ $(\mathrm{m}, 5 \mathrm{H}) ;{ }^{13} \mathrm{C}$ NMR $\left(62.9 \mathrm{MHz}, \mathrm{CDCl}_{3}\right) \delta(\mathrm{ppm})$ 14.2, 22.8, 26.1, 29.3, 29.4, 29.5, 31.9, $34.3,38.8,45.7,51.7,57.3,83.9,126.8,128.2,128.4,137.3,169.9,173.4 ; M S(E I) \mathrm{m} / \mathrm{z}$ $\% 377\left(\mathrm{M}^{+}\right)$.

(S)-1-Phenylethyl-(R)-2-methoxy-2-phenylacetamide [(R)-22]

$[\alpha]_{\mathrm{D}}=-212.5\left(\mathrm{c}=0.006, \mathrm{CH}_{3} \mathrm{Cl}\right) ;{ }^{1} \mathrm{H} \mathrm{NMR}\left(250.13 \mathrm{MHz}, \mathrm{CH}_{3} \mathrm{CN}-d_{3}\right) \delta(\mathrm{ppm}) 1.45(\mathrm{~d}$, $\mathrm{J}=7.04 \mathrm{~Hz}, 3 \mathrm{H}), 3.33(\mathrm{~s}, 3 \mathrm{H}), 4.64(\mathrm{~s}, 1 \mathrm{H}), 4.97$ (m, 1H), 7.14-7.38 (m, 10H), 7.43 (br, $1 \mathrm{H}) ;{ }^{13} \mathrm{C}$ NMR (62.9 MHz, $\left.\mathrm{CH}_{3} \mathrm{CN}-d_{3}\right) \delta(\mathrm{ppm}) 21.3,47.9,56.2,83.3,125.5$,

126.5, 126.8, 127.8, 128.0, 128.0, 137.6, 144.0, 160.0; MS (EI) m/z \% $269\left(\mathrm{M}^{+}\right)$.

(S)-1-Phenylethyl-(S)-2-methoxy-2-phenylacetamide [(S)-22]

$[\alpha]_{\mathrm{D}}=-4.74\left(\mathrm{c}=0.005, \mathrm{CH}_{3} \mathrm{Cl}\right) ;{ }^{1} \mathrm{H} \mathrm{NMR}\left(250.13 \mathrm{MHz}, \mathrm{CH}_{3} \mathrm{CN}-d_{3}\right) \delta(\mathrm{ppm}) 1.42(\mathrm{~d}$, $\mathrm{J}=7.02 \mathrm{~Hz}, 3 \mathrm{H}), 3.26(\mathrm{~s}, 3 \mathrm{H}), 4.58(\mathrm{~s}, 1 \mathrm{H}), 4.97(\mathrm{~m}, 1 \mathrm{H}), 7.25(\mathrm{~m}, 1 \mathrm{H}), 7.29-7.44(\mathrm{~m}$, $10 \mathrm{H}) ;{ }^{13} \mathrm{C}$ NMR $\left(62.9 \mathrm{MHz}, \mathrm{CH}_{3} \mathrm{CN}-d_{3}\right) \delta(\mathrm{ppm}) 21.1,47.9,56.3,83.2,125.7,126.6$, 127.0, 127.9, 128.0, 128.1, 137.6, 144.0, 169.1; MS (EI) m/z \% $269\left(\mathrm{M}^{+}\right)$.

( $S$ ) - Methyl 2-[( $R$ )-2-methoxy-2-phenylacetamido]-3-[1-methyl-1H-indol-3yl]propanoate $[(R)-24]$

$[\alpha]_{\mathrm{D}}=-127.65\left(\mathrm{c}=0.0009, \mathrm{CH}_{3} \mathrm{Cl}\right) ;{ }^{1} \mathrm{H}$ NMR $\left(250.13 \mathrm{MHz}, \mathrm{CH}_{3} \mathrm{CN}-d_{3}\right) \delta(\mathrm{ppm}) 3.20$ (s, 3H), $3.28(\mathrm{dd}, \mathrm{J}=6.48,5.09 \mathrm{~Hz}, 2 \mathrm{H}), 3.64(\mathrm{~s}, 3 \mathrm{H}), 3.75(\mathrm{~s}, 3 \mathrm{H}), 4.56(\mathrm{~s}, 1 \mathrm{H}), 4.69$ (m, 
1H), $6.97(\mathrm{~s}, 1 \mathrm{H}), 7.09$ (dd, J=7.17, $7.17 \mathrm{~Hz}, 1 \mathrm{H}), 7.21(\mathrm{dd}, \mathrm{J}=7.23,7.23 \mathrm{~Hz}, 1 \mathrm{H})$, 7.28-7.41 (m, 7H), $7.55(\mathrm{~d}, \mathrm{~J}=7.88 \mathrm{~Hz}, 1 \mathrm{H}) ;{ }^{13} \mathrm{C} \mathrm{NMR}\left(62.9 \mathrm{MHz}, \mathrm{CH}_{3} \mathrm{CN}-d_{3}\right) \delta(\mathrm{ppm})$ $26.5,31.8,51.5,52.3,56.3,83.0,109.2,118.2,118.6,121.2,127.2,127.5,127.8,127.9$, 128.0, 137.3, 169.7, 171.6; MS (EI) m/z \% $380\left(\mathrm{M}^{+}\right)$. 


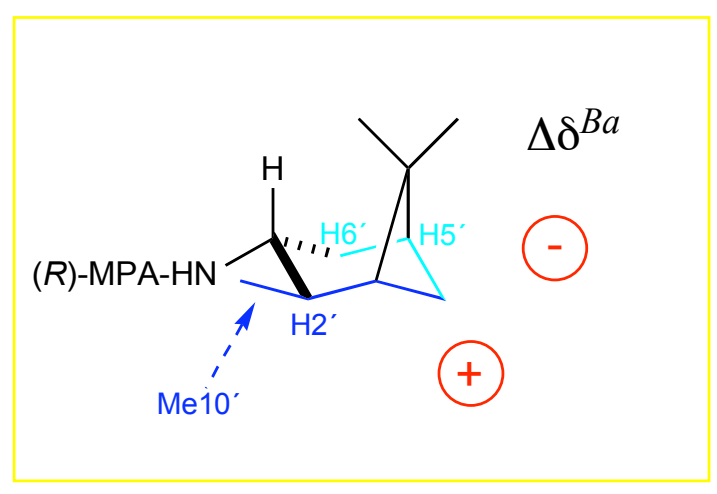

a)
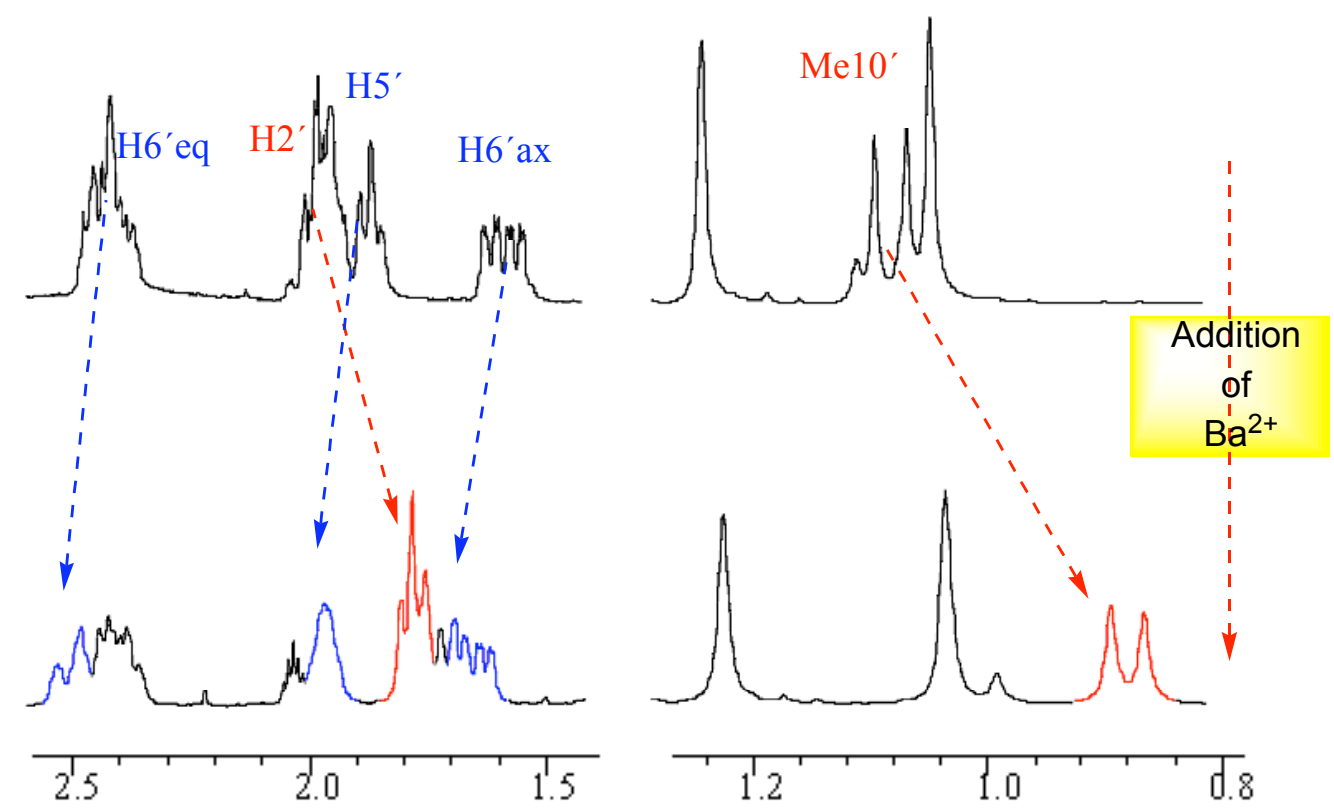

Figure 1-SI. a) Partial ${ }^{1} \mathrm{H}$ NMR spectrum $(250 \mathrm{MHz})$ of the (R)-MPA amide of (-)isopinocampheylamine without barium(II). b) Idem with barium(II). 

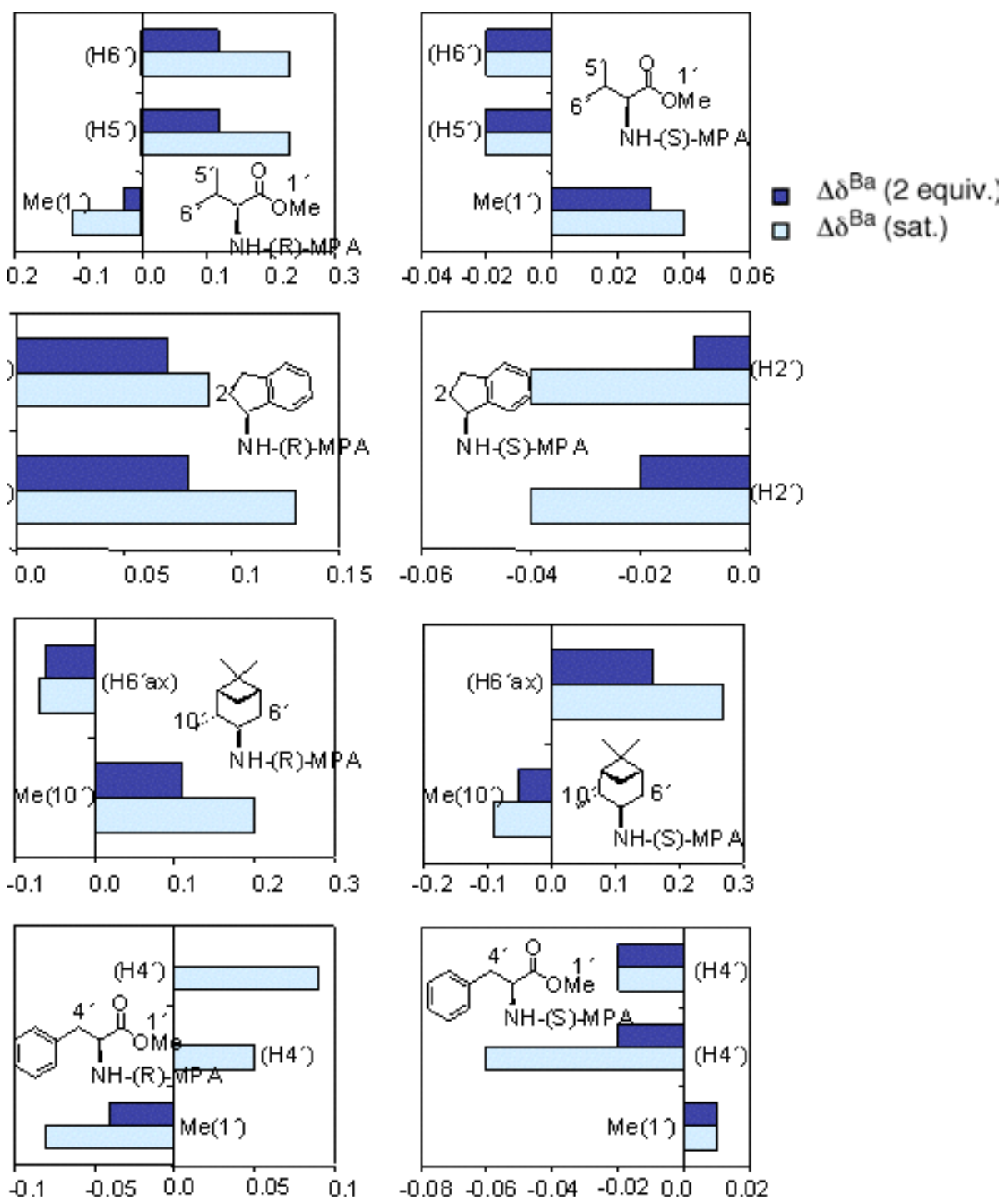

Figure 2-SI. Representation of the $\Delta \delta^{B a}$ values obtained by addition of 2 equivalents of $\mathrm{Ba}\left(\mathrm{ClO}_{4}\right)_{2}$ versus those obtained on saturation of $\mathrm{Ba}\left(\mathrm{ClO}_{4}\right)_{2}$. 


\title{
GEOMETRIES AND TOTAL ENERGIES CALCULATED IN THIS WORK WITH GAUSSIAN98
}

\author{
Structure: (AP, ac, ap) \\ Calculation: gas-phase b3lyp/LanL2DZ \\ Total energy $=-672.6291807$ hartree \\ Cartesian coordinates:
}

\begin{tabular}{|c|c|c|c|c|c|}
\hline \multirow{2}{*}{$\begin{array}{l}\text { Center } \\
\text { Number }\end{array}$} & \multirow{2}{*}{$\begin{array}{l}\text { Atomic } \\
\text { Number }\end{array}$} & \multirow{2}{*}{$\begin{array}{l}\text { Atomic } \\
\text { Type }\end{array}$} & \multicolumn{3}{|c|}{ Coordinates (Angstroms) } \\
\hline & & & $\mathrm{x}$ & $\mathrm{Y}$ & Z \\
\hline 1 & 8 & 0 & -0.129465 & 1.978716 & -0.228176 \\
\hline 2 & 6 & 0 & 0.267439 & 0.919564 & 0.696840 \\
\hline 3 & 6 & 0 & 0.743383 & 3.147096 & -0.224015 \\
\hline 4 & 6 & 0 & -0.938424 & -0.034726 & 0.873602 \\
\hline 5 & 6 & 0 & 1.504814 & 0.160819 & 0.219938 \\
\hline 6 & 1 & 0 & 0.462077 & 1.347225 & 1.692969 \\
\hline 7 & 1 & 0 & 1.763849 & 2.881111 & -0.529982 \\
\hline 8 & 1 & 0 & 0.769250 & 3.609257 & 0.774329 \\
\hline 9 & 1 & 0 & 0.314197 & 3.850653 & -0.942070 \\
\hline 10 & 8 & 0 & -0.911440 & -0.926163 & 1.764739 \\
\hline 11 & 7 & 0 & -1.978511 & 0.173486 & 0.019583 \\
\hline 12 & 6 & 0 & 2.395533 & -0.398616 & 1.157306 \\
\hline 13 & 6 & 0 & 1.745595 & -0.019577 & -1.158262 \\
\hline 14 & 6 & 0 & 3.515353 & -1.132428 & 0.723527 \\
\hline 15 & 6 & 0 & -3.200797 & -0.657499 & 0.032250 \\
\hline 16 & 6 & 0 & 2.867671 & -0.748059 & -1.592604 \\
\hline 17 & 1 & 0 & -1.894461 & 0.958713 & -0.619773 \\
\hline 18 & 1 & 0 & 2.203434 & -0.278065 & 2.220887 \\
\hline 19 & 1 & 0 & 1.058396 & 0.415127 & -1.879714 \\
\hline 20 & 6 & 0 & 3.755802 & -1.307701 & -0.652908 \\
\hline 21 & 6 & 0 & -4.374813 & 0.158652 & -0.538660 \\
\hline 22 & 6 & 0 & -2.976741 & -1.978684 & -0.737414 \\
\hline 23 & 1 & 0 & 4.194507 & -1.565137 & 1.454319 \\
\hline 24 & 1 & 0 & -3.400742 & -0.906061 & 1.082258 \\
\hline 25 & 1 & 0 & 3.047727 & -0.881844 & -2.656985 \\
\hline 26 & 1 & 0 & 4.622099 & -1.873064 & -0.988678 \\
\hline 27 & 1 & 0 & -5.297156 & -0.433401 & -0.508143 \\
\hline 28 & 1 & 0 & -4.537171 & 1.077402 & 0.038769 \\
\hline 29 & 1 & 0 & -4.190297 & 0.434409 & -1.587124 \\
\hline 30 & 1 & 0 & -2.774747 & -1.782631 & -1.799010 \\
\hline 31 & 1 & 0 & -3.866361 & -2.618543 & -0.666661 \\
\hline 32 & 1 & 0 & -2.126678 & -2.523028 & -0.312589 \\
\hline
\end{tabular}


Structure: (AP, ap, ap)

Calculation: gas-phase b3lyp/LanL2DZ

Total energy $=-672.6292019$ hartree

Cartesian coordinates:

\begin{tabular}{|c|c|c|c|c|c|}
\hline \multirow{2}{*}{$\begin{array}{l}\text { Center } \\
\text { Number }\end{array}$} & \multirow{2}{*}{$\begin{array}{l}\text { Atomic } \\
\text { Number }\end{array}$} & \multirow{2}{*}{$\begin{array}{c}\text { Atomic } \\
\text { Type }\end{array}$} & \multicolumn{3}{|c|}{ Coordinates (Angstroms) } \\
\hline & & & $\mathrm{x}$ & $\mathrm{Y}$ & $\mathrm{z}$ \\
\hline 1 & 8 & 0 & 0.115434 & 1.922353 & 0.143593 \\
\hline 2 & 6 & 0 & -0.263607 & 0.800500 & -0.712598 \\
\hline 3 & 6 & 0 & -0.732276 & 3.101665 & 0.010666 \\
\hline 4 & 6 & 0 & 0.925819 & -0.189058 & -0.750445 \\
\hline 5 & 6 & 0 & -1.540003 & 0.107870 & -0.237810 \\
\hline 6 & 8 & 0 & 0.936591 & -1.130597 & -1.588509 \\
\hline 7 & 7 & 0 & 1.901502 & 0.036878 & 0.173173 \\
\hline 8 & 6 & 0 & -2.408624 & -0.490570 & -1.171746 \\
\hline 9 & 6 & 0 & -1.840170 & 0.027941 & 1.138251 \\
\hline 10 & 6 & 0 & -3.565226 & -1.164254 & -0.736660 \\
\hline 11 & 6 & 0 & 3.106140 & -0.810754 & 0.287739 \\
\hline 12 & 6 & 0 & -2.998815 & -0.640115 & 1.573413 \\
\hline 13 & 6 & 0 & -3.864641 & -1.239382 & 0.637227 \\
\hline 14 & 6 & 0 & 4.184928 & -0.383567 & -0.733747 \\
\hline 15 & 6 & 0 & 3.625216 & -0.764353 & 1.736849 \\
\hline 16 & 1 & 0 & -0.400524 & 1.150168 & -1.747759 \\
\hline 17 & 1 & 0 & -1.769631 & 2.878771 & 0.293256 \\
\hline 18 & 1 & 0 & -0.707906 & 3.483318 & -1.021206 \\
\hline 19 & 1 & 0 & -0.318020 & 3.852394 & 0.688562 \\
\hline 20 & 1 & 0 & 1.811277 & 0.879025 & 0.734991 \\
\hline 21 & 1 & 0 & -2.171618 & -0.446818 & -2.232244 \\
\hline 22 & 1 & 0 & -1.170155 & 0.492775 & 1.857052 \\
\hline 23 & 1 & 0 & -4.227091 & -1.627900 & -1.464322 \\
\hline 24 & 1 & 0 & 2.789704 & -1.831795 & 0.042456 \\
\hline 25 & 1 & 0 & -3.224600 & -0.696730 & 2.635975 \\
\hline 26 & 1 & 0 & -4.759174 & -1.758263 & 0.973990 \\
\hline 27 & 1 & 0 & 5.051474 & -1.055799 & -0.679654 \\
\hline 28 & 1 & 0 & 3.779872 & -0.425904 & -1.750565 \\
\hline 29 & 1 & 0 & 4.529741 & 0.640140 & -0.533970 \\
\hline 30 & 1 & 0 & 3.932099 & 0.254630 & 2.014511 \\
\hline 31 & 1 & 0 & 4.500080 & -1.416335 & 1.846528 \\
\hline 32 & 1 & 0 & 2.855312 & -1.099411 & 2.442676 \\
\hline
\end{tabular}


Structure: (AP, sp, ap)

Calculation: gas-phase b3lyp/LanL2DZ

Total energy $=-672.6264586$ hartree

Cartesian coordinates:

\begin{tabular}{|c|c|c|c|c|c|}
\hline \multirow{2}{*}{$\begin{array}{l}\text { Center } \\
\text { Number }\end{array}$} & \multirow{2}{*}{$\begin{array}{l}\text { Atomic } \\
\text { Number }\end{array}$} & \multirow{2}{*}{$\begin{array}{c}\text { Atomic } \\
\text { Type }\end{array}$} & \multicolumn{3}{|c|}{ Coordinates (Angstroms) } \\
\hline & & & $\mathrm{x}$ & $\mathrm{Y}$ & $\mathrm{z}$ \\
\hline 1 & 8 & 0 & 0.010500 & 2.061114 & -0.327410 \\
\hline 2 & 6 & 0 & 0.221620 & 0.950014 & 0.598018 \\
\hline 3 & 6 & 0 & 0.951484 & 3.165662 & -0.179442 \\
\hline 4 & 6 & 0 & -1.053618 & 0.067959 & 0.583603 \\
\hline 5 & 6 & 0 & 1.460810 & 0.127408 & 0.249316 \\
\hline 6 & 1 & 0 & 0.312807 & 1.331946 & 1.626716 \\
\hline 7 & 1 & 0 & 1.981083 & 2.838620 & -0.375952 \\
\hline 8 & 1 & 0 & 0.890261 & 3.595049 & 0.831837 \\
\hline 9 & 1 & 0 & 0.655968 & 3.918038 & -0.915204 \\
\hline 10 & 8 & 0 & -1.199888 & -0.826568 & 1.460151 \\
\hline 11 & 7 & 0 & -1.931579 & 0.345277 & -0.420180 \\
\hline 12 & 6 & 0 & 2.204798 & -0.497824 & 1.269304 \\
\hline 13 & 6 & 0 & 1.848496 & -0.044731 & -1.096197 \\
\hline 14 & 6 & 0 & 3.324527 & -1.288430 & 0.949769 \\
\hline 15 & 6 & 0 & -3.209804 & -0.373853 & -0.652411 \\
\hline 16 & 6 & 0 & 2.970452 & -0.830216 & -1.415977 \\
\hline 17 & 1 & 0 & -1.693771 & 1.147812 & -0.996542 \\
\hline 18 & 1 & 0 & 1.898894 & -0.382568 & 2.306520 \\
\hline 19 & 1 & 0 & 1.274683 & 0.440918 & -1.881484 \\
\hline 20 & 6 & 0 & 3.711583 & -1.455543 & -0.393766 \\
\hline 21 & 6 & 0 & -2.965153 & -1.867214 & -0.964347 \\
\hline 22 & 6 & 0 & -4.189470 & -0.181645 & 0.527896 \\
\hline 23 & 1 & 0 & 3.890115 & -1.771155 & 1.743264 \\
\hline 24 & 1 & 0 & -3.645444 & 0.098846 & -1.544490 \\
\hline 25 & 1 & 0 & 3.264292 & -0.957320 & -2.455548 \\
\hline 26 & 1 & 0 & 4.577730 & -2.065024 & -0.641111 \\
\hline 27 & 1 & 0 & -3.916964 & -2.361039 & -1.199439 \\
\hline 28 & 1 & 0 & -2.293757 & -1.980937 & -1.824680 \\
\hline 29 & 1 & 0 & -2.513805 & -2.366461 & -0.101643 \\
\hline 30 & 1 & 0 & -3.775292 & -0.624833 & 1.438678 \\
\hline 31 & 1 & 0 & -5.147408 & -0.668812 & 0.303614 \\
\hline 32 & 1 & 0 & -4.377592 & 0.884075 & 0.710201 \\
\hline
\end{tabular}


Structure: (AP, ac, $-\mathrm{SC})$

Calculation: gas-phase b3lyp/LanL2DZ

Total energy $=-672.6280916$ hartree

Cartesian coordinates:

\begin{tabular}{|c|c|c|c|c|c|}
\hline \multirow{2}{*}{$\begin{array}{l}\text { Center } \\
\text { Number }\end{array}$} & \multirow{2}{*}{$\begin{array}{l}\text { Atomic } \\
\text { Number }\end{array}$} & \multirow{2}{*}{$\begin{array}{c}\text { Atomic } \\
\text { Type }\end{array}$} & \multicolumn{3}{|c|}{ Coordinates (Angstroms) } \\
\hline & & & $\mathrm{x}$ & $\mathrm{Y}$ & $\mathrm{z}$ \\
\hline 1 & 8 & 0 & -0.046155 & 2.068817 & -0.363897 \\
\hline 2 & 6 & 0 & 0.294663 & 1.075415 & 0.648112 \\
\hline 3 & 6 & 0 & -0.805379 & 3.204395 & 0.153010 \\
\hline 4 & 6 & 0 & -0.892864 & 0.109761 & 0.902250 \\
\hline 5 & 6 & 0 & 1.535681 & 0.302753 & 0.197444 \\
\hline 6 & 1 & 0 & 0.498888 & 1.567694 & 1.611396 \\
\hline 7 & 1 & 0 & -0.221158 & 3.751607 & 0.906629 \\
\hline 8 & 1 & 0 & -1.759220 & 2.881251 & 0.595555 \\
\hline 9 & 1 & 0 & -0.999656 & 3.855205 & -0.703358 \\
\hline 10 & 8 & 0 & -0.998541 & -0.510900 & 1.995535 \\
\hline 11 & 7 & 0 & -1.760539 & -0.015794 & -0.142663 \\
\hline 12 & 6 & 0 & 2.069322 & -0.699735 & 1.037265 \\
\hline 13 & 6 & 0 & 2.158486 & 0.581933 & -1.034976 \\
\hline 14 & 6 & 0 & 3.215023 & -1.413796 & 0.644635 \\
\hline 15 & 6 & 0 & -2.902650 & -0.955329 & -0.132139 \\
\hline 16 & 6 & 0 & 3.305813 & -0.136520 & -1.424922 \\
\hline 17 & 1 & 0 & -1.559054 & 0.529871 & -0.976033 \\
\hline 18 & 1 & 0 & 1.580316 & -0.925602 & 1.981712 \\
\hline 19 & 1 & 0 & 1.747706 & 1.359954 & -1.669011 \\
\hline 20 & 6 & 0 & 3.838317 & -1.134918 & -0.588204 \\
\hline 21 & 6 & 0 & -3.989049 & -0.447346 & -1.097184 \\
\hline 22 & 6 & 0 & -2.438533 & -2.388985 & -0.475383 \\
\hline 23 & 1 & 0 & 3.619433 & -2.183665 & 1.297746 \\
\hline 24 & 1 & 0 & -3.291759 & -0.959132 & 0.893607 \\
\hline 25 & 1 & 0 & 3.782194 & 0.086034 & -2.377256 \\
\hline 26 & 1 & 0 & 4.725485 & -1.687022 & -0.890108 \\
\hline 27 & 1 & 0 & -4.854148 & -1.120679 & -1.081516 \\
\hline 28 & 1 & 0 & -4.331282 & 0.557037 & -0.817624 \\
\hline 29 & 1 & 0 & -3.613769 & -0.412133 & -2.130262 \\
\hline 30 & 1 & 0 & -2.035059 & -2.435719 & -1.495667 \\
\hline 31 & 1 & 0 & -3.280180 & -3.090409 & -0.403598 \\
\hline 32 & 1 & 0 & -1.660379 & -2.712831 & 0.223869 \\
\hline
\end{tabular}


Structure: ( $A P,-a c,-s c)$

Calculation: gas-phase b3lyp/LanL2DZ

Total energy $=-672.6279699$ hartree

Cartesian coordinates:

\begin{tabular}{|c|c|c|c|c|c|}
\hline \multirow{2}{*}{$\begin{array}{l}\text { Center } \\
\text { Number }\end{array}$} & \multirow{2}{*}{$\begin{array}{l}\text { Atomic } \\
\text { Number }\end{array}$} & \multirow{2}{*}{$\begin{array}{c}\text { Atomic } \\
\text { Type }\end{array}$} & \multicolumn{3}{|c|}{ Coordinates (Angstroms) } \\
\hline & & & $\mathrm{x}$ & $\mathrm{Y}$ & $\mathrm{z}$ \\
\hline 1 & 8 & 0 & 0.016947 & 1.964520 & 0.487881 \\
\hline 2 & 6 & 0 & -0.309369 & 1.025962 & -0.580108 \\
\hline 3 & 6 & 0 & 0.835589 & 3.093673 & 0.054183 \\
\hline 4 & 6 & 0 & 0.860323 & 0.036189 & -0.824186 \\
\hline 5 & 6 & 0 & -1.590448 & 0.274069 & -0.215015 \\
\hline 6 & 8 & 0 & 1.008807 & -0.527866 & -1.942783 \\
\hline 7 & 7 & 0 & 1.654380 & -0.186132 & 0.262324 \\
\hline 8 & 6 & 0 & -2.111396 & -0.682842 & -1.113969 \\
\hline 9 & 6 & 0 & -2.262200 & 0.527819 & 0.997023 \\
\hline 10 & 6 & 0 & -3.292379 & -1.377879 & -0.799425 \\
\hline 11 & 6 & 0 & 2.776118 & -1.149586 & 0.256904 \\
\hline 12 & 6 & 0 & -3.444897 & -0.171254 & 1.308489 \\
\hline 13 & 6 & 0 & -3.964246 & -1.124799 & 0.413265 \\
\hline 14 & 6 & 0 & 4.050461 & -0.515692 & -0.344919 \\
\hline 15 & 6 & 0 & 3.003640 & -1.670829 & 1.687746 \\
\hline 16 & 1 & 0 & -0.457077 & 1.565335 & -1.528310 \\
\hline 17 & 1 & 0 & 0.307745 & 3.686940 & -0.706130 \\
\hline 18 & 1 & 0 & 1.801024 & 2.757307 & -0.352339 \\
\hline 19 & 1 & 0 & 1.005125 & 3.705589 & 0.943817 \\
\hline 20 & 1 & 0 & 1.442566 & 0.343065 & 1.103663 \\
\hline 21 & 1 & 0 & -1.586105 & -0.889132 & -2.043343 \\
\hline 22 & 1 & 0 & -1.860678 & 1.270862 & 1.677220 \\
\hline 23 & 1 & 0 & -3.686294 & -2.112966 & -1.497480 \\
\hline 24 & 1 & 0 & 2.468539 & -1.978532 & -0.392558 \\
\hline 25 & 1 & 0 & -3.958662 & 0.031223 & 2.245759 \\
\hline 26 & 1 & 0 & -4.878543 & -1.662179 & 0.654780 \\
\hline 27 & 1 & 0 & 4.857449 & -1.258102 & -0.400069 \\
\hline 28 & 1 & 0 & 3.849998 & -0.151743 & -1.358475 \\
\hline 29 & 1 & 0 & 4.398066 & 0.324638 & 0.271491 \\
\hline 30 & 1 & 0 & 3.296873 & -0.854701 & 2.364136 \\
\hline 31 & 1 & 0 & 3.809355 & -2.414373 & 1.695469 \\
\hline 32 & 1 & 0 & 2.096747 & -2.142521 & 2.085388 \\
\hline
\end{tabular}


Structure: (AP, sp, $-\mathrm{sc})$

Calculation: gas-phase b3lyp/LanL2DZ

Total energy $=-672.6252488$ hartree

Cartesian coordinates:

\begin{tabular}{|c|c|c|c|c|c|}
\hline \multirow{2}{*}{$\begin{array}{l}\text { Center } \\
\text { Number }\end{array}$} & \multirow{2}{*}{$\begin{array}{l}\text { Atomic } \\
\text { Number }\end{array}$} & \multirow{2}{*}{$\begin{array}{c}\text { Atomic } \\
\text { Type }\end{array}$} & \multicolumn{3}{|c|}{ Coordinates (Angstroms) } \\
\hline & & & $\mathrm{x}$ & $\mathrm{Y}$ & $\mathrm{z}$ \\
\hline 1 & 8 & 0 & 0.069937 & 2.122170 & -0.348728 \\
\hline 2 & 6 & 0 & 0.247954 & 1.031148 & 0.603078 \\
\hline 3 & 6 & 0 & -0.737373 & 3.227804 & 0.160651 \\
\hline 4 & 6 & 0 & -0.983657 & 0.083161 & 0.602353 \\
\hline 5 & 6 & 0 & 1.524725 & 0.264169 & 0.254185 \\
\hline 6 & 1 & 0 & 0.324769 & 1.427926 & 1.626971 \\
\hline 7 & 1 & 0 & -0.261032 & 3.683395 & 1.040481 \\
\hline 8 & 1 & 0 & -1.751525 & 2.894929 & 0.427020 \\
\hline 9 & 1 & 0 & -0.792018 & 3.961614 & -0.647529 \\
\hline 10 & 8 & 0 & -1.254013 & -0.612051 & 1.620104 \\
\hline 11 & 7 & 0 & -1.677394 & 0.067228 & -0.572122 \\
\hline 12 & 6 & 0 & 1.918392 & -0.828786 & 1.057752 \\
\hline 13 & 6 & 0 & 2.317130 & 0.635948 & -0.849788 \\
\hline 14 & 6 & 0 & 3.092781 & -1.540593 & 0.756099 \\
\hline 15 & 6 & 0 & -2.841225 & -0.808900 & -0.865187 \\
\hline 16 & 6 & 0 & 3.492597 & -0.080341 & -1.148983 \\
\hline 17 & 1 & 0 & -1.331406 & 0.693515 & -1.293741 \\
\hline 18 & 1 & 0 & 1.300905 & -1.125173 & 1.902175 \\
\hline 19 & 1 & 0 & 2.014220 & 1.483148 & -1.455063 \\
\hline 20 & 6 & 0 & 3.884950 & -1.169361 & -0.348748 \\
\hline 21 & 6 & 0 & -2.445996 & -2.301702 & -0.809302 \\
\hline 22 & 6 & 0 & -4.035027 & -0.489943 & 0.062937 \\
\hline 23 & 1 & 0 & 3.388229 & -2.380598 & 1.380531 \\
\hline 24 & 1 & 0 & -3.130575 & -0.565100 & -1.897407 \\
\hline 25 & 1 & 0 & 4.099816 & 0.214423 & -2.002119 \\
\hline 26 & 1 & 0 & 4.793977 & -1.719993 & -0.580032 \\
\hline 27 & 1 & 0 & -3.302335 & -2.926749 & -1.093667 \\
\hline 28 & 1 & 0 & -1.618592 & -2.512216 & -1.498099 \\
\hline 29 & 1 & 0 & -2.134450 & -2.573849 & 0.203764 \\
\hline 30 & 1 & 0 & -3.772546 & -0.699325 & 1.104407 \\
\hline 31 & 1 & 0 & -4.898968 & -1.108285 & -0.212770 \\
\hline 32 & 1 & 0 & -4.325607 & 0.565157 & -0.021373 \\
\hline
\end{tabular}


Structure: (AP, ac, ap)

Calculation: Onsager b3lyp/LanL2Dz

Total energy $=-672.6306024$ hartree

Cartesian coordinates:

\begin{tabular}{|c|c|c|c|c|c|}
\hline \multirow{2}{*}{$\begin{array}{l}\text { Center } \\
\text { Number }\end{array}$} & \multirow{2}{*}{$\begin{array}{l}\text { Atomic } \\
\text { Number }\end{array}$} & \multirow{2}{*}{$\begin{array}{l}\text { Atomic } \\
\text { Type }\end{array}$} & \multicolumn{3}{|c|}{ Coordinates (Angstroms) } \\
\hline & & & $\mathrm{x}$ & Y & Z \\
\hline-1 & -1 & & - & -1 & ---------- \\
\hline 1 & 8 & 0 & -0.136601 & 1.963634 & -0.223489 \\
\hline 2 & 6 & 0 & 0.266165 & 0.909327 & 0.701383 \\
\hline 3 & 6 & 0 & 0.732366 & 3.137040 & -0.235317 \\
\hline 4 & 6 & 0 & -0.938800 & -0.046947 & 0.877945 \\
\hline 5 & 6 & 0 & 1.505390 & 0.154324 & 0.222613 \\
\hline 6 & 1 & 0 & 0.461913 & 1.337587 & 1.697258 \\
\hline 7 & 1 & 0 & 1.749862 & 2.873540 & -0.551903 \\
\hline 8 & 1 & 0 & 0.765898 & 3.604510 & 0.760017 \\
\hline 9 & 1 & 0 & 0.291874 & 3.833656 & -0.952554 \\
\hline 10 & 8 & 0 & -0.912530 & -0.944815 & 1.764819 \\
\hline 11 & 7 & 0 & -1.979053 & 0.168883 & 0.027353 \\
\hline 12 & 6 & 0 & 2.412830 & -0.382813 & 1.156769 \\
\hline 13 & 6 & 0 & 1.736389 & -0.035932 & -1.155973 \\
\hline 14 & 6 & 0 & 3.539611 & -1.104135 & 0.719736 \\
\hline 15 & 6 & 0 & -3.206073 & -0.653463 & 0.034302 \\
\hline 16 & 6 & 0 & 2.864924 & -0.752012 & -1.593819 \\
\hline 17 & 1 & 0 & -1.891040 & 0.959699 & -0.605566 \\
\hline 18 & 1 & 0 & 2.231262 & -0.249102 & 2.220800 \\
\hline 19 & 1 & 0 & 1.040075 & 0.386657 & -1.875686 \\
\hline 20 & 6 & 0 & 3.769971 & -1.289246 & -0.657151 \\
\hline 21 & 6 & 0 & -4.374055 & 0.173673 & -0.533434 \\
\hline 22 & 6 & 0 & -2.989521 & -1.971072 & -0.744309 \\
\hline 23 & 1 & 0 & 4.235556 & -1.514401 & 1.448069 \\
\hline 24 & 1 & 0 & -3.408776 & -0.907384 & 1.082797 \\
\hline 25 & 1 & 0 & 3.039847 & -0.888003 & -2.658592 \\
\hline 26 & 1 & 0 & 4.644662 & -1.839314 & -0.996727 \\
\hline 27 & 1 & 0 & -5.300904 & -0.411831 & -0.508618 \\
\hline 28 & 1 & 0 & -4.530827 & 1.090385 & 0.048434 \\
\hline 29 & 1 & 0 & -4.186311 & 0.455213 & -1.579352 \\
\hline 30 & 1 & 0 & -2.784639 & -1.767977 & -1.803730 \\
\hline 31 & 1 & 0 & -3.884319 & -2.604926 & -0.680681 \\
\hline 32 & 1 & 0 & -2.142910 & -2.523506 & -0.322539 \\
\hline
\end{tabular}


Structure: (AP, ap, ap)

Calculation: Onsager b3lyp/LanL2Dz

Total energy $=-672.6306232$ hartree

Cartesian coordinates:

\begin{tabular}{|c|c|c|c|c|c|}
\hline \multirow{2}{*}{$\begin{array}{l}\text { Center } \\
\text { Number }\end{array}$} & \multirow{2}{*}{$\begin{array}{l}\text { Atomic } \\
\text { Number }\end{array}$} & \multirow{2}{*}{$\begin{array}{l}\text { Atomic } \\
\text { Type }\end{array}$} & \multicolumn{3}{|c|}{ Coordinates (Angstroms) } \\
\hline & & & $\mathrm{x}$ & Y & Z \\
\hline-1 & -7 & & 10 & -- & -------- \\
\hline 1 & 8 & 0 & 0.122867 & 1.907182 & 0.120034 \\
\hline 2 & 6 & 0 & -0.261731 & 0.780396 & -0.723815 \\
\hline 3 & 6 & 0 & -0.724229 & 3.089636 & -0.007577 \\
\hline 4 & 6 & 0 & 0.926716 & -0.211535 & -0.749286 \\
\hline 5 & 6 & 0 & -1.540498 & 0.097658 & -0.240521 \\
\hline 6 & 8 & 0 & 0.936114 & -1.171391 & -1.568700 \\
\hline 7 & 7 & 0 & 1.905383 & 0.036035 & 0.164267 \\
\hline 8 & 6 & 0 & -2.428739 & -0.481615 & -1.167789 \\
\hline 9 & 6 & 0 & -1.828796 & 0.017709 & 1.138149 \\
\hline 10 & 6 & 0 & -3.593084 & -1.136228 & -0.723950 \\
\hline 11 & 6 & 0 & 3.113614 & -0.802311 & 0.296387 \\
\hline 12 & 6 & 0 & -2.994600 & -0.631396 & 1.582325 \\
\hline 13 & 6 & 0 & -3.880245 & -1.211441 & 0.652525 \\
\hline 14 & 6 & 0 & 4.187137 & -0.394828 & -0.739076 \\
\hline 15 & 6 & 0 & 3.637320 & -0.715050 & 1.742073 \\
\hline 16 & 1 & 0 & -0.399180 & 1.118793 & -1.762894 \\
\hline 17 & 1 & 0 & -1.757896 & 2.870830 & 0.289881 \\
\hline 18 & 1 & 0 & -0.710491 & 3.466439 & -1.041150 \\
\hline 19 & 1 & 0 & -0.299022 & 3.840962 & 0.662065 \\
\hline 20 & 1 & 0 & 1.810649 & 0.889312 & 0.709481 \\
\hline 21 & 1 & 0 & -2.204099 & -0.431941 & -2.230908 \\
\hline 22 & 1 & 0 & -1.147582 & 0.473193 & 1.852233 \\
\hline 23 & 1 & 0 & -4.273925 & -1.579662 & -1.447073 \\
\hline 24 & 1 & 0 & 2.801815 & -1.831220 & 0.078202 \\
\hline 25 & 1 & 0 & -3.213528 & -0.682512 & 2.646411 \\
\hline 26 & 1 & 0 & -4.783663 & -1.709673 & 0.996804 \\
\hline 27 & 1 & 0 & 5.058542 & -1.059770 & -0.669659 \\
\hline 28 & 1 & 0 & 3.778899 & -0.464037 & -1.753335 \\
\hline 29 & 1 & 0 & 4.525869 & 0.635358 & -0.565702 \\
\hline 30 & 1 & 0 & 3.936591 & 0.312716 & 1.992735 \\
\hline 31 & 1 & 0 & 4.517945 & -1.356797 & 1.866344 \\
\hline 32 & 1 & 0 & 2.872359 & -1.036531 & 2.459342 \\
\hline
\end{tabular}




\begin{abstract}
Structure: (AP, sp, ap)
Calculation: Onsager b3lyp/LanL2Dz

Total energy $=-672.6278842$ hartree

Cartesian coordinates:
\end{abstract}

\begin{tabular}{|c|c|c|c|c|c|}
\hline \multirow{2}{*}{$\begin{array}{l}\text { Center } \\
\text { Number }\end{array}$} & \multirow{2}{*}{$\begin{array}{l}\text { Atomic } \\
\text { Number }\end{array}$} & \multirow{2}{*}{$\begin{array}{c}\text { Atomic } \\
\text { Type }\end{array}$} & \multicolumn{3}{|c|}{ Coordinates (Angstroms) } \\
\hline & & & $\mathrm{x}$ & $\mathrm{Y}$ & Z \\
\hline 1 & 8 & 0 & 0.002920 & 2.043507 & -0.341884 \\
\hline 2 & 6 & 0 & 0.221245 & 0.945425 & 0.594545 \\
\hline 3 & 6 & 0 & 0.953173 & 3.147266 & -0.233464 \\
\hline 4 & 6 & 0 & -1.054668 & 0.063949 & 0.590543 \\
\hline 5 & 6 & 0 & 1.460682 & 0.121574 & 0.249482 \\
\hline 6 & 1 & 0 & 0.316811 & 1.337600 & 1.619211 \\
\hline 7 & 1 & 0 & 1.975687 & 2.812441 & -0.450395 \\
\hline 8 & 1 & 0 & 0.916680 & 3.592101 & 0.772012 \\
\hline 9 & 1 & 0 & 0.642592 & 3.888404 & -0.973708 \\
\hline 10 & 8 & 0 & -1.201574 & -0.829864 & 1.469755 \\
\hline 11 & 7 & 0 & -1.935543 & 0.342701 & -0.409674 \\
\hline 12 & 6 & 0 & 2.246303 & -0.442633 & 1.273415 \\
\hline 13 & 6 & 0 & 1.814339 & -0.101810 & -1.098021 \\
\hline 14 & 6 & 0 & 3.373704 & -1.223643 & 0.956480 \\
\hline 15 & 6 & 0 & -3.217304 & -0.367976 & -0.638549 \\
\hline 16 & 6 & 0 & 2.943297 & -0.877644 & -1.415776 \\
\hline 17 & 1 & 0 & -1.694029 & 1.144260 & -0.987064 \\
\hline 18 & 1 & 0 & 1.971406 & -0.281287 & 2.313433 \\
\hline 19 & 1 & 0 & 1.213514 & 0.342890 & -1.887251 \\
\hline 20 & 6 & 0 & 3.726259 & -1.441990 & -0.389135 \\
\hline 21 & 6 & 0 & -2.982563 & -1.863026 & -0.951215 \\
\hline 22 & 6 & 0 & -4.193339 & -0.169452 & 0.544007 \\
\hline 23 & 1 & 0 & 3.975971 & -1.653838 & 1.753468 \\
\hline 24 & 1 & 0 & -3.652818 & 0.106514 & -1.529265 \\
\hline 25 & 1 & 0 & 3.214070 & -1.038694 & -2.456723 \\
\hline 26 & 1 & 0 & 4.601943 & -2.038117 & -0.635537 \\
\hline 27 & 1 & 0 & -3.938298 & -2.351289 & -1.183011 \\
\hline 28 & 1 & 0 & -2.315819 & -1.979309 & -1.814645 \\
\hline 29 & 1 & 0 & -2.528893 & -2.363593 & -0.090264 \\
\hline 30 & 1 & 0 & -3.778074 & -0.613041 & 1.454321 \\
\hline 31 & 1 & 0 & -5.154916 & -0.650918 & 0.321878 \\
\hline 32 & 1 & 0 & -4.374575 & 0.897591 & 0.724407 \\
\hline
\end{tabular}


Structure: (AP, ac, $-\mathrm{SC})$

Calculation: Onsager b3lyp/LanL2DZ

Total energy $=-672.6292905$ hartree

Cartesian coordinates:

\begin{tabular}{|c|c|c|c|c|c|}
\hline \multirow{2}{*}{$\begin{array}{l}\text { Center } \\
\text { Number }\end{array}$} & \multirow{2}{*}{$\begin{array}{l}\text { Atomic } \\
\text { Number }\end{array}$} & \multirow{2}{*}{$\begin{array}{l}\text { Atomic } \\
\text { Type }\end{array}$} & \multicolumn{3}{|c|}{ Coordinates (Angstroms) } \\
\hline & & & $\mathrm{x}$ & Y & Z \\
\hline-1 & -1 & & - & -- & --------- \\
\hline 1 & 8 & 0 & -0.050269 & 2.048969 & -0.341895 \\
\hline 2 & 6 & 0 & 0.295138 & 1.047037 & 0.657350 \\
\hline 3 & 6 & 0 & -0.792221 & 3.192487 & 0.186318 \\
\hline 4 & 6 & 0 & -0.884217 & 0.066252 & 0.896546 \\
\hline 5 & 6 & 0 & 1.545855 & 0.292823 & 0.200791 \\
\hline 6 & 1 & 0 & 0.492508 & 1.527806 & 1.628125 \\
\hline 7 & 1 & 0 & -0.190685 & 3.734980 & 0.929436 \\
\hline 8 & 1 & 0 & -1.741930 & 2.877760 & 0.643071 \\
\hline 9 & 1 & 0 & -0.993732 & 3.843548 & -0.667678 \\
\hline 10 & 8 & 0 & -0.961491 & -0.599906 & 1.966915 \\
\hline 11 & 7 & 0 & -1.774660 & -0.015157 & -0.130825 \\
\hline 12 & 6 & 0 & 2.088533 & -0.716657 & 1.026453 \\
\hline 13 & 6 & 0 & 2.168693 & 0.597071 & -1.025762 \\
\hline 14 & 6 & 0 & 3.243196 & -1.411837 & 0.625814 \\
\hline 15 & 6 & 0 & -2.929397 & -0.939756 & -0.130687 \\
\hline 16 & 6 & 0 & 3.324772 & -0.102491 & -1.423889 \\
\hline 17 & 1 & 0 & -1.592564 & 0.568240 & -0.943314 \\
\hline 18 & 1 & 0 & 1.596081 & -0.963295 & 1.963803 \\
\hline 19 & 1 & 0 & 1.749405 & 1.378659 & -1.649573 \\
\hline 20 & 6 & 0 & 3.866649 & -1.107361 & -0.600983 \\
\hline 21 & 6 & 0 & -4.034491 & -0.374908 & -1.041423 \\
\hline 22 & 6 & 0 & -2.493829 & -2.361532 & -0.552060 \\
\hline 23 & 1 & 0 & 3.653458 & -2.188687 & 1.267609 \\
\hline 24 & 1 & 0 & -3.290201 & -0.984897 & 0.904462 \\
\hline 25 & 1 & 0 & 3.798895 & 0.139297 & -2.372513 \\
\hline 26 & 1 & 0 & 4.759996 & -1.645485 & -0.910647 \\
\hline 27 & 1 & 0 & -4.909111 & -1.035743 & -1.030763 \\
\hline 28 & 1 & 0 & -4.354530 & 0.620514 & -0.709454 \\
\hline 29 & 1 & 0 & -3.688704 & -0.299201 & -2.082279 \\
\hline 30 & 1 & 0 & -2.119704 & -2.365288 & -1.584376 \\
\hline 31 & 1 & 0 & -3.343543 & -3.054177 & -0.490650 \\
\hline 32 & 1 & 0 & -1.701021 & -2.727207 & 0.109558 \\
\hline
\end{tabular}


Structure: ( $A P,-a c,-s c)$

Calculation: Onsager b3lyp/LanL2DZ

Total energy $=-672.6291613$ hartree

Cartesian coordinates:

\begin{tabular}{|c|c|c|c|c|c|}
\hline \multirow{2}{*}{$\begin{array}{l}\text { Center } \\
\text { Number }\end{array}$} & \multirow{2}{*}{$\begin{array}{l}\text { Atomic } \\
\text { Number }\end{array}$} & \multirow{2}{*}{$\begin{array}{l}\text { Atomic } \\
\text { Type }\end{array}$} & \multicolumn{3}{|c|}{ Coordinates (Angstroms) } \\
\hline & & & $\mathrm{x}$ & Y & Z \\
\hline-1 & -7 & & 10 & -- & -------- \\
\hline 1 & 8 & 0 & 0.021841 & 1.955016 & 0.438887 \\
\hline 2 & 6 & 0 & -0.308300 & 0.991403 & -0.602692 \\
\hline 3 & 6 & 0 & 0.827225 & 3.084527 & -0.022217 \\
\hline 4 & 6 & 0 & 0.853554 & -0.015559 & -0.818222 \\
\hline 5 & 6 & 0 & -1.597550 & 0.261663 & -0.220087 \\
\hline 6 & 8 & 0 & 0.974145 & -0.638037 & -1.910555 \\
\hline 7 & 7 & 0 & 1.669547 & -0.179788 & 0.259829 \\
\hline 8 & 6 & 0 & -2.125806 & -0.718000 & -1.089897 \\
\hline 9 & 6 & 0 & -2.269685 & 0.558878 & 0.981962 \\
\hline 10 & 6 & 0 & -3.314362 & -1.391332 & -0.756685 \\
\hline 11 & 6 & 0 & 2.804856 & -1.127856 & 0.282077 \\
\hline 12 & 6 & 0 & -3.459768 & -0.118516 & 1.312319 \\
\hline 13 & 6 & 0 & -3.986784 & -1.094137 & 0.445761 \\
\hline 14 & 6 & 0 & 4.061021 & -0.503188 & -0.366099 \\
\hline 15 & 6 & 0 & 3.060652 & -1.577003 & 1.732570 \\
\hline 16 & 1 & 0 & -0.450084 & 1.504800 & -1.566392 \\
\hline 17 & 1 & 0 & 0.284668 & 3.662133 & -0.784067 \\
\hline 18 & 1 & 0 & 1.790244 & 2.749168 & -0.434665 \\
\hline 19 & 1 & 0 & 1.002419 & 3.710296 & 0.856129 \\
\hline 20 & 1 & 0 & 1.474206 & 0.395493 & 1.075017 \\
\hline 21 & 1 & 0 & -1.597039 & -0.959509 & -2.008694 \\
\hline 22 & 1 & 0 & -1.860929 & 1.317141 & 1.640532 \\
\hline 23 & 1 & 0 & -3.712812 & -2.145764 & -1.431795 \\
\hline 24 & 1 & 0 & 2.499503 & -1.990093 & -0.323708 \\
\hline 25 & 1 & 0 & -3.971455 & 0.117325 & 2.242757 \\
\hline 26 & 1 & 0 & -4.906218 & -1.615497 & 0.703365 \\
\hline 27 & 1 & 0 & 4.879344 & -1.234439 & -0.395935 \\
\hline 28 & 1 & 0 & 3.843207 & -0.188995 & -1.392820 \\
\hline 29 & 1 & 0 & 4.403094 & 0.369427 & 0.206235 \\
\hline 30 & 1 & 0 & 3.350219 & -0.725483 & 2.364817 \\
\hline 31 & 1 & 0 & 3.879078 & -2.305771 & 1.764373 \\
\hline 32 & 1 & 0 & 2.167519 & -2.042833 & 2.166656 \\
\hline
\end{tabular}


Structure: (AP, sp, $-\mathrm{sc})$

Calculation: Onsager b3lyp/LanL2Dz

Total energy $=-672.6263664$ hartree

Cartesian coordinates:

\begin{tabular}{|c|c|c|c|c|c|}
\hline \multirow{2}{*}{$\begin{array}{l}\text { Center } \\
\text { Number }\end{array}$} & \multirow{2}{*}{$\begin{array}{l}\text { Atomic } \\
\text { Number }\end{array}$} & \multirow{2}{*}{$\begin{array}{l}\text { Atomic } \\
\text { Type }\end{array}$} & \multicolumn{3}{|c|}{ Coordinates (Angstroms) } \\
\hline & & & $\mathrm{x}$ & Y & Z \\
\hline$-1-5>$ & $-1,4$ & & t & -- & -------- \\
\hline 1 & 8 & 0 & 0.072247 & 2.106127 & -0.339838 \\
\hline 2 & 6 & 0 & 0.249579 & 1.010969 & 0.604503 \\
\hline 3 & 6 & 0 & -0.707949 & 3.228012 & 0.179994 \\
\hline 4 & 6 & 0 & -0.979456 & 0.058716 & 0.595630 \\
\hline 5 & 6 & 0 & 1.530967 & 0.250896 & 0.254361 \\
\hline 6 & 1 & 0 & 0.323391 & 1.400749 & 1.631573 \\
\hline 7 & 1 & 0 & -0.208493 & 3.677289 & 1.050002 \\
\hline 8 & 1 & 0 & -1.723372 & 2.913634 & 0.462779 \\
\hline 9 & 1 & 0 & -0.762377 & 3.959838 & -0.629478 \\
\hline 10 & 8 & 0 & -1.223744 & -0.674512 & 1.594878 \\
\hline 11 & 7 & 0 & -1.698995 & 0.085609 & -0.561007 \\
\hline 12 & 6 & 0 & 1.921010 & -0.856296 & 1.040181 \\
\hline 13 & 6 & 0 & 2.332882 & 0.646498 & -0.834486 \\
\hline 14 & 6 & 0 & 3.101017 & -1.557863 & 0.735872 \\
\hline 15 & 6 & 0 & -2.879651 & -0.766890 & -0.856897 \\
\hline 16 & 6 & 0 & 3.513715 & -0.059573 & -1.136488 \\
\hline 17 & 1 & 0 & -1.369165 & 0.740120 & -1.265586 \\
\hline 18 & 1 & 0 & 1.293785 & -1.171425 & 1.870417 \\
\hline 19 & 1 & 0 & 2.031100 & 1.502793 & -1.427130 \\
\hline 20 & 6 & 0 & 3.902656 & -1.162460 & -0.353741 \\
\hline 21 & 6 & 0 & -2.499952 & -2.264688 & -0.863231 \\
\hline 22 & 6 & 0 & -4.047745 & -0.467200 & 0.109283 \\
\hline 23 & 1 & 0 & 3.392743 & -2.410158 & 1.345938 \\
\hline 24 & 1 & 0 & -3.189945 & -0.483754 & -1.872361 \\
\hline 25 & 1 & 0 & 4.126234 & 0.253698 & -1.979015 \\
\hline 26 & 1 & 0 & 4.815598 & -1.705490 & -0.588581 \\
\hline 27 & 1 & 0 & -3.369593 & -2.869224 & -1.151513 \\
\hline 28 & 1 & 0 & -1.690854 & -2.458140 & -1.578336 \\
\hline 29 & 1 & 0 & -2.168885 & -2.575943 & 0.132447 \\
\hline 30 & 1 & 0 & -3.765495 & -0.717402 & 1.136661 \\
\hline 31 & 1 & 0 & -4.926148 & -1.062959 & -0.170062 \\
\hline 32 & 1 & 0 & -4.326021 & 0.593470 & 0.069082 \\
\hline
\end{tabular}


Structure: (SP, ac, sc)

Calculation: Onsager b3lyp/LanL2Dz

Total energy $=-672.6215347$ hartree

Cartesian coordinates:

\begin{tabular}{|c|c|c|c|c|c|}
\hline \multirow{2}{*}{$\begin{array}{l}\text { Center } \\
\text { Number }\end{array}$} & \multirow{2}{*}{$\begin{array}{l}\text { Atomic } \\
\text { Number }\end{array}$} & \multirow{2}{*}{$\begin{array}{l}\text { Atomic } \\
\text { Type }\end{array}$} & \multicolumn{3}{|c|}{ Coordinates (Angstroms) } \\
\hline & & & $\mathrm{x}$ & Y & Z \\
\hline-1 & -1 & & ----- & $-\ldots$ & ---------- \\
\hline 1 & 8 & 0 & 0.307457 & -0.943052 & -2.334479 \\
\hline 2 & 6 & 0 & 0.547665 & -0.661864 & -0.933034 \\
\hline 3 & 6 & 0 & 1.283075 & -0.357775 & -3.249869 \\
\hline 4 & 6 & 0 & 0.493379 & 0.868619 & -0.638959 \\
\hline 5 & 6 & 0 & -0.517767 & -1.389526 & -0.127923 \\
\hline 6 & 1 & 0 & 1.547810 & -1.032898 & -0.644406 \\
\hline 7 & 1 & 0 & 2.307300 & -0.675978 & -2.995188 \\
\hline 8 & 1 & 0 & 1.218905 & 0.737233 & -3.244548 \\
\hline 9 & 1 & 0 & 1.022568 & -0.738275 & -4.241138 \\
\hline 10 & 8 & 0 & -0.204606 & 1.662676 & -1.322687 \\
\hline 11 & 7 & 0 & 1.255030 & 1.267511 & 0.428158 \\
\hline 12 & 6 & 0 & -0.162694 & -2.247957 & 0.930940 \\
\hline 13 & 6 & 0 & -1.881046 & -1.190117 & -0.434827 \\
\hline 14 & 6 & 0 & -1.157805 & -2.898651 & 1.686183 \\
\hline 15 & 6 & 0 & 1.262394 & 2.661051 & 0.935912 \\
\hline 16 & 6 & 0 & -2.873694 & -1.842751 & 0.315899 \\
\hline 17 & 1 & 0 & 0.887129 & -2.424068 & 1.160975 \\
\hline 18 & 1 & 0 & -2.144713 & -0.531001 & -1.256740 \\
\hline 19 & 6 & 0 & -2.516325 & -2.696062 & 1.380390 \\
\hline 20 & 6 & 0 & 0.040517 & 2.923316 & 1.844334 \\
\hline 21 & 6 & 0 & 2.595716 & 2.932285 & 1.655699 \\
\hline 22 & 1 & 0 & -0.874088 & -3.559952 & 2.501254 \\
\hline 23 & 1 & 0 & 1.181054 & 3.306328 & 0.053080 \\
\hline 24 & 1 & 0 & -3.923355 & -1.685694 & 0.076634 \\
\hline 25 & 1 & 0 & -3.286181 & -3.197261 & 1.962820 \\
\hline 26 & 1 & 0 & 0.022494 & 3.973503 & 2.163410 \\
\hline 27 & 1 & 0 & -0.888063 & 2.709739 & 1.303959 \\
\hline 28 & 1 & 0 & 0.077806 & 2.292870 & 2.742608 \\
\hline 29 & 1 & 0 & 2.712943 & 2.281667 & 2.534534 \\
\hline 30 & 1 & 0 & 2.630726 & 3.969165 & 2.009145 \\
\hline 31 & 1 & 0 & 3.450311 & 2.770572 & 0.987269 \\
\hline 32 & 1 & 0 & 1.768841 & 0.571088 & 0.958087 \\
\hline
\end{tabular}


Structure: (SC, -ac, sc)

Calculation: Onsager b3lyp/LanL2DZ

Total energy $=-672.6214054$ hartree

Cartesian coordinates:

\begin{tabular}{|c|c|c|c|c|c|}
\hline \multirow{2}{*}{$\begin{array}{l}\text { Center } \\
\text { Number }\end{array}$} & \multirow{2}{*}{$\begin{array}{l}\text { Atomic } \\
\text { Number }\end{array}$} & \multirow{2}{*}{$\begin{array}{c}\text { Atomic } \\
\text { Type }\end{array}$} & \multicolumn{3}{|c|}{ Coordinates (Angstroms) } \\
\hline & & & $\mathrm{X}$ & $\mathrm{Y}$ & Z \\
\hline & & & & & \\
\hline 1 & 8 & 0 & -0.620855 & 2.353391 & -0.311654 \\
\hline 2 & 6 & 0 & -0.296163 & 0.968764 & -0.594641 \\
\hline 3 & 6 & 0 & 0.449184 & 3.301578 & -0.606618 \\
\hline 4 & 6 & 0 & 0.918505 & 0.483010 & 0.253144 \\
\hline 5 & 6 & 0 & -1.525390 & 0.131882 & -0.277363 \\
\hline 6 & 1 & 0 & -0.038737 & 0.858070 & -1.663826 \\
\hline 7 & 1 & 0 & 0.768034 & 3.226748 & -1.659134 \\
\hline 8 & 1 & 0 & 1.309425 & 3.143935 & 0.056116 \\
\hline 9 & 1 & 0 & 0.023465 & 4.292451 & -0.427685 \\
\hline 10 & 8 & 0 & 1.150147 & 0.920710 & 1.410717 \\
\hline 11 & 7 & 0 & 1.685029 & -0.477287 & -0.352704 \\
\hline 12 & 6 & 0 & -2.043923 & -0.776315 & -1.220934 \\
\hline 13 & 6 & 0 & -2.143987 & 0.252945 & 0.985552 \\
\hline 14 & 6 & 0 & -3.167018 & -1.566474 & -0.907664 \\
\hline 15 & 6 & 0 & 2.829662 & -1.138078 & 0.320673 \\
\hline 16 & 6 & 0 & -3.267202 & -0.532389 & 1.296335 \\
\hline 17 & 1 & 0 & -1.584419 & -0.862174 & -2.204471 \\
\hline 18 & 1 & 0 & -1.738184 & 0.958380 & 1.704410 \\
\hline 19 & 6 & 0 & -3.780394 & -1.446615 & 0.353128 \\
\hline 20 & 6 & 0 & 3.027134 & -2.543684 & -0.275743 \\
\hline 21 & 6 & 0 & 4.105519 & -0.273286 & 0.216473 \\
\hline 22 & 1 & 0 & -3.560522 & -2.264626 & -1.642505 \\
\hline 23 & 1 & 0 & 2.555821 & -1.220597 & 1.379642 \\
\hline 24 & 1 & 0 & -3.740359 & -0.437045 & 2.271418 \\
\hline 25 & 1 & 0 & -4.647322 & -2.056791 & 0.596851 \\
\hline 26 & 1 & 0 & 3.847814 & -3.058859 & 0.236388 \\
\hline 27 & 1 & 0 & 2.121324 & -3.153053 & -0.168581 \\
\hline 28 & 1 & 0 & 3.288444 & -2.489882 & -1.342503 \\
\hline 29 & 1 & 0 & 4.413395 & -0.153126 & -0.830621 \\
\hline 30 & 1 & 0 & 4.931444 & -0.746680 & 0.763087 \\
\hline 31 & 1 & 0 & 3.929594 & 0.717608 & 0.648994 \\
\hline 32 & 1 & 0 & 1.455686 & -0.774185 & -1.295208 \\
\hline
\end{tabular}


Structure: (SC, sp, sc)

Calculation: Onsager b3lyp/LanL2Dz

Total energy $=-672.6183338$ hartree

Cartesian coordinates:

\begin{tabular}{|c|c|c|c|c|c|}
\hline \multirow{2}{*}{$\begin{array}{l}\text { Center } \\
\text { Number }\end{array}$} & \multirow{2}{*}{$\begin{array}{l}\text { Atomic } \\
\text { Number }\end{array}$} & \multirow{2}{*}{$\begin{array}{l}\text { Atomic } \\
\text { Type }\end{array}$} & \multicolumn{3}{|c|}{ Coordinates (Angstroms) } \\
\hline & & & $\mathrm{x}$ & Y & Z \\
\hline-1 & -1 & & -1 & -- & -------- \\
\hline 1 & 8 & 0 & -0.449863 & 2.377936 & 0.100645 \\
\hline 2 & 6 & 0 & -0.243987 & 1.081426 & -0.513788 \\
\hline 3 & 6 & 0 & 0.639423 & 3.328424 & -0.102841 \\
\hline 4 & 6 & 0 & 1.013679 & 0.365477 & 0.073909 \\
\hline 5 & 6 & 0 & -1.489124 & 0.246829 & -0.258743 \\
\hline 6 & 1 & 0 & -0.096194 & 1.204454 & -1.602236 \\
\hline 7 & 1 & 0 & 0.842199 & 3.476238 & -1.176206 \\
\hline 8 & 1 & 0 & 1.552355 & 2.997359 & 0.407824 \\
\hline 9 & 1 & 0 & 0.294741 & 4.270033 & 0.332657 \\
\hline 10 & 8 & 0 & 1.390815 & 0.549546 & 1.260710 \\
\hline 11 & 7 & 0 & 1.636403 & -0.481126 & -0.806210 \\
\hline 12 & 6 & 0 & -2.154677 & -0.400473 & -1.318058 \\
\hline 13 & 6 & 0 & -1.974167 & 0.103846 & 1.059073 \\
\hline 14 & 6 & 0 & -3.291599 & -1.193237 & -1.067563 \\
\hline 15 & 6 & 0 & 2.794835 & -1.357800 & -0.472686 \\
\hline 16 & 6 & 0 & -3.111038 & -0.683763 & 1.308315 \\
\hline 17 & 1 & 0 & -1.800004 & -0.278597 & -2.340477 \\
\hline 18 & 1 & 0 & -1.456539 & 0.611315 & 1.867645 \\
\hline 19 & 6 & 0 & -3.771381 & -1.337455 & 0.247419 \\
\hline 20 & 6 & 0 & 4.040798 & -0.525527 & -0.097534 \\
\hline 21 & 6 & 0 & 2.426381 & -2.384501 & 0.621260 \\
\hline 22 & 1 & 0 & -3.799462 & -1.688352 & -1.891684 \\
\hline 23 & 1 & 0 & 3.013189 & -1.904673 & -1.400546 \\
\hline 24 & 1 & 0 & -3.481081 & -0.792211 & 2.325654 \\
\hline 25 & 1 & 0 & -4.648934 & -1.949816 & 0.442563 \\
\hline 26 & 1 & 0 & 4.898775 & -1.191852 & 0.058020 \\
\hline 27 & 1 & 0 & 4.294873 & 0.179971 & -0.898400 \\
\hline 28 & 1 & 0 & 3.859991 & 0.037082 & 0.823289 \\
\hline 29 & 1 & 0 & 2.180993 & -1.872398 & 1.556715 \\
\hline 30 & 1 & 0 & 3.274460 & -3.057846 & 0.799622 \\
\hline 31 & 1 & 0 & 1.564186 & -2.989889 & 0.315418 \\
\hline 32 & 1 & 0 & 1.249150 & -0.562268 & -1.739802 \\
\hline
\end{tabular}




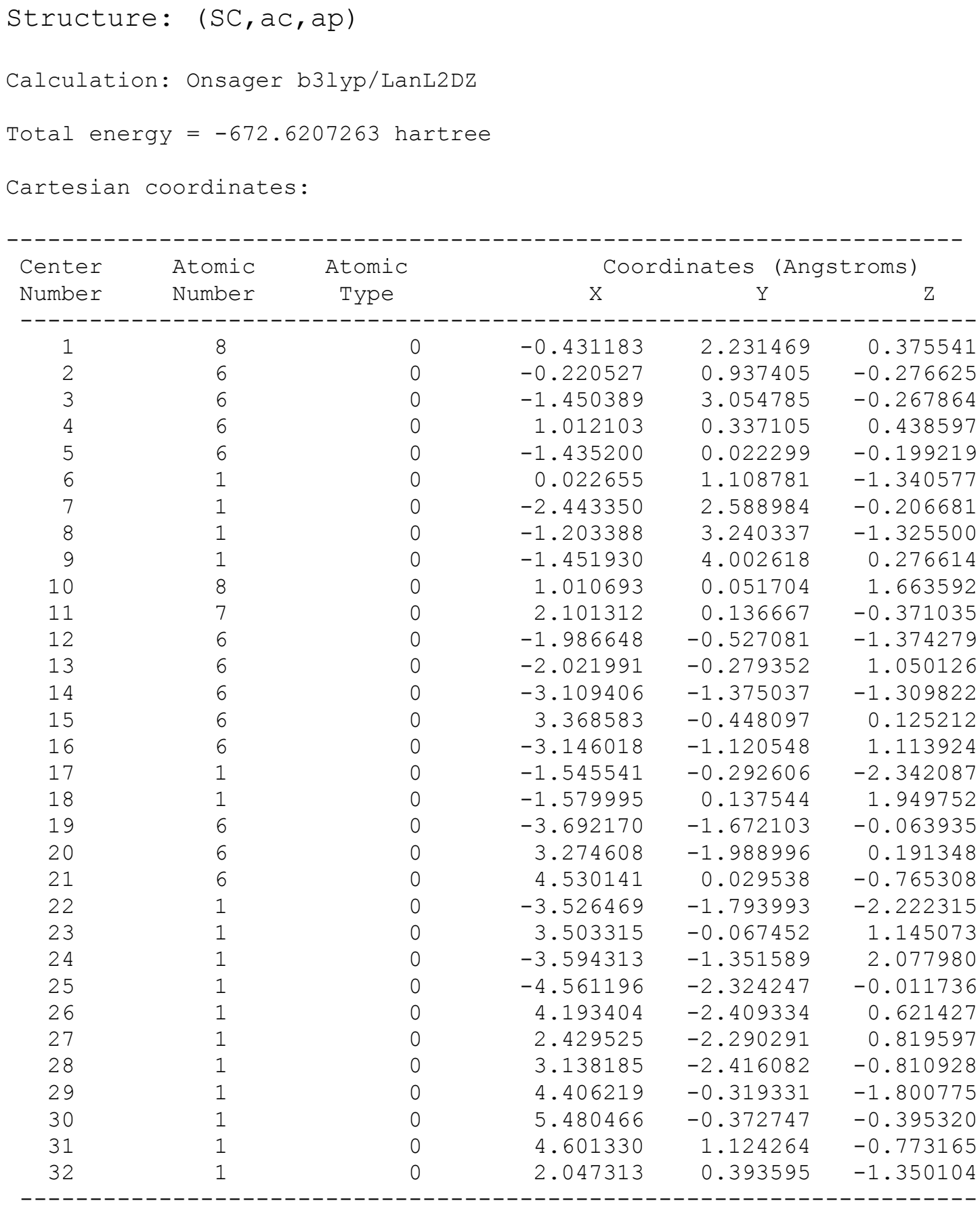


Structure: (SC,-ac, ap)

Calculation: Onsager b3lyp/LanL2DZ

Total energy $=-672.6209078$ hartree

Cartesian coordinates:

\begin{tabular}{|c|c|c|c|c|c|}
\hline \multirow{2}{*}{$\begin{array}{l}\text { Center } \\
\text { Number }\end{array}$} & \multirow{2}{*}{$\begin{array}{l}\text { Atomic } \\
\text { Number }\end{array}$} & \multirow{2}{*}{$\begin{array}{l}\text { Atomic } \\
\text { Type }\end{array}$} & \multicolumn{3}{|c|}{ Coordinates (Angstroms) } \\
\hline & & & $\mathrm{x}$ & Y & Z \\
\hline-1 & -7 & & - & -1 & --------- \\
\hline 1 & 8 & 0 & -0.279096 & 2.137866 & -0.017363 \\
\hline 2 & 6 & 0 & -0.210939 & 0.735817 & -0.439965 \\
\hline 3 & 6 & 0 & -1.236140 & 2.936004 & -0.777647 \\
\hline 4 & 6 & 0 & 0.988554 & 0.158613 & 0.346770 \\
\hline 5 & 6 & 0 & -1.500949 & -0.030034 & -0.184468 \\
\hline 6 & 1 & 0 & 0.010622 & 0.699249 & -1.521559 \\
\hline 7 & 1 & 0 & -2.265660 & 2.585174 & -0.624309 \\
\hline 8 & 1 & 0 & -1.004455 & 2.916052 & -1.854607 \\
\hline 9 & 1 & 0 & -1.133351 & 3.957547 & -0.402569 \\
\hline 10 & 8 & 0 & 0.977473 & 0.027921 & 1.597750 \\
\hline 11 & 7 & 0 & 2.057716 & -0.208685 & -0.430599 \\
\hline 12 & 6 & 0 & -2.135704 & -0.721339 & -1.236147 \\
\hline 13 & 6 & 0 & -2.076643 & -0.048880 & 1.105584 \\
\hline 14 & 6 & 0 & -3.330971 & -1.430688 & -1.008399 \\
\hline 15 & 6 & 0 & 3.305166 & -0.765668 & 0.140145 \\
\hline 16 & 6 & 0 & -3.272506 & -0.751821 & 1.331610 \\
\hline 17 & 1 & 0 & -1.702341 & -0.705878 & -2.235201 \\
\hline 18 & 1 & 0 & -1.572178 & 0.474656 & 1.911453 \\
\hline 19 & 6 & 0 & -3.902558 & -1.445538 & 0.277392 \\
\hline 20 & 6 & 0 & 3.992089 & -1.657519 & -0.910447 \\
\hline 21 & 6 & 0 & 4.229721 & 0.363020 & 0.649227 \\
\hline 22 & 1 & 0 & -3.812051 & -1.960598 & -1.826901 \\
\hline 23 & 1 & 0 & 2.999907 & -1.374157 & 0.999954 \\
\hline 24 & 1 & 0 & -3.711843 & -0.764837 & 2.326878 \\
\hline 25 & 1 & 0 & -4.827399 & -1.989914 & 0.455224 \\
\hline 26 & 1 & 0 & 4.903633 & -2.101016 & -0.492830 \\
\hline 27 & 1 & 0 & 3.331964 & -2.471798 & -1.233393 \\
\hline 28 & 1 & 0 & 4.285241 & -1.075521 & -1.796387 \\
\hline 29 & 1 & 0 & 4.559714 & 1.002440 & -0.180423 \\
\hline 30 & 1 & 0 & 5.120726 & -0.061065 & 1.131120 \\
\hline 31 & 1 & 0 & 3.700689 & 0.982818 & 1.381025 \\
\hline 32 & 1 & 0 & 2.031032 & -0.030447 & -1.428320 \\
\hline
\end{tabular}




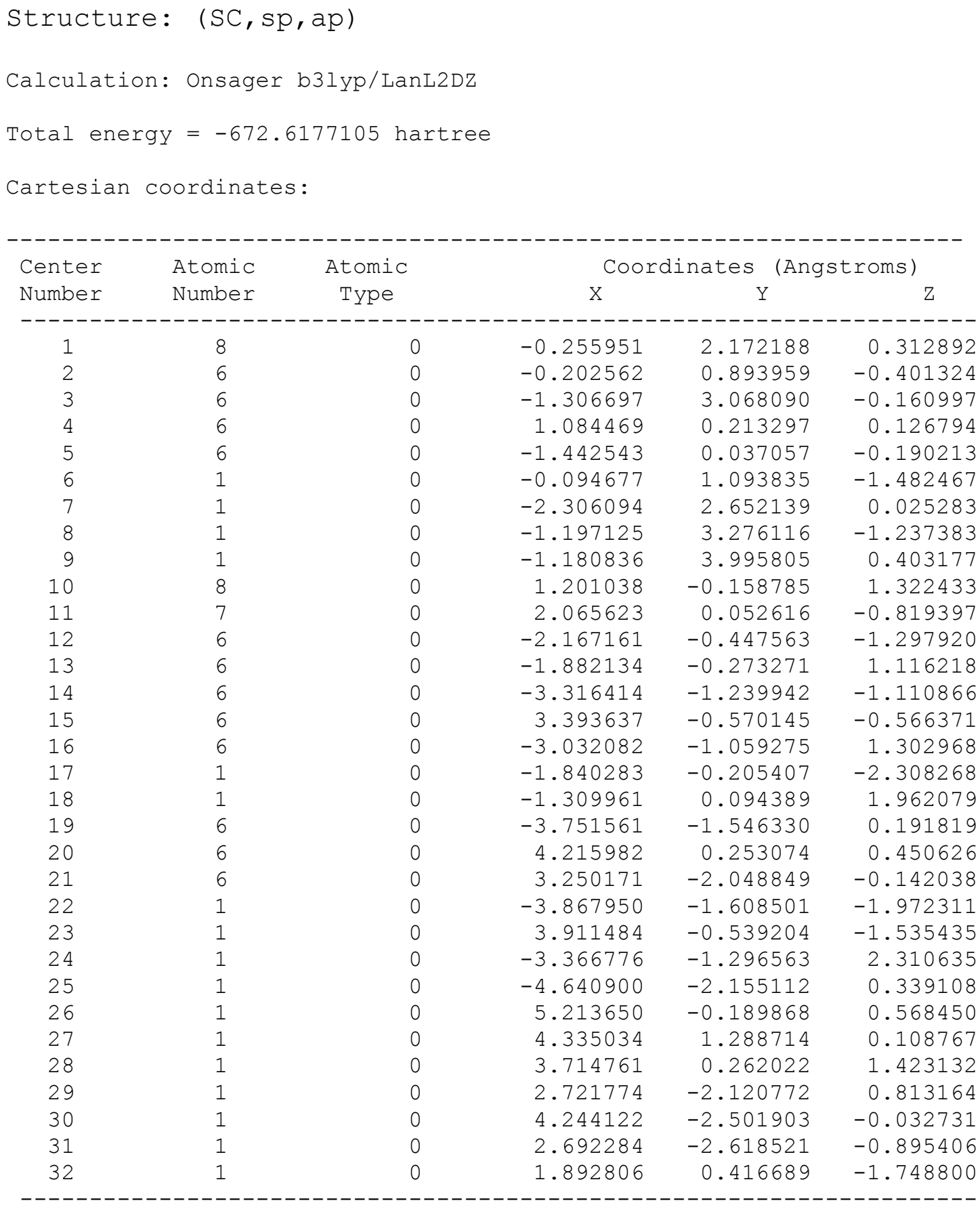


Structure: (AP, ac, ap)

Calculation: IPCM b3lyp/LanL2DZ using the Onsager geometry Total energy $=-672.6496987$ hartree 
Structure: (AP, ap, ap)

Calculation: IPCM b3lyp/LanL2Dz using the Onsager geometry Total energy $=-672.648831$ hartree 
Structure: (AP, sp, ap)

Calculation: IPCM b3lyp/LanL2Dz using the Onsager geometry Total energy $=-672.644347$ hartree 
Structure: (AP, ac, $-\mathrm{SC})$

Calculation: IPCM b3lyp/LanL2Dz using the Onsager geometry Total energy $=-672.644728$ hartree 
Structure: (AP, -ac, -sc)

Calculation: IPCM b3lyp/LanL2DZ using the Onsager geometry Total energy $=-672.645090$ hartree 
Structure: (AP, sp, -sc)

Calculation: IPCM b3lyp/LanL2Dz using the Onsager geometry Total energy $=-672.642876$ hartree 
Structure: (SP, ac, sc)

Calculation: IPCM b3lyp/LanL2Dz using the Onsager geometry Total energy $=-672.648040$ hartree 
Structure: $(\mathrm{SC},-\mathrm{ac}, \mathrm{SC})$

Calculation: IPCM b3lyp/LanL2DZ using the onsager geometry Total energy $=-672.646803$ hartree 
Structure: (SC, sp, sc)

Calculation: IPCM b3lyp/LanL2Dz using the Onsager geometry Total energy $=-672.646400$ hartree 
Structure: (SC, ac, ap)

Calculation: IPCM b3lyp/LanL2DZ using the Onsager geometry Total energy $=-672.642993$ hartree 
Structure: (SC, $-\mathrm{ac}, \mathrm{ap})$

Calculation: IPCM b3lyp/LanL2Dz using the Onsager geometry Total energy $=-672.646260$ hartree 
Structure: (SC, sp, ap)

Calculation: IPCM b3lyp/LanL2DZ using the Onsager geometry Total energy $=-672.639097$ hartree 


\begin{abstract}
Structure: (SP, ap_1- $\left.\mathrm{Ba}^{2+}\right)$
Calculation: gas-phase b3lyp/LanL2DZ

Total energy $=-697.438301$ hartree

Cartesian coordinates:
\end{abstract}

\begin{tabular}{|c|c|c|c|c|c|}
\hline \multirow{2}{*}{$\begin{array}{l}\text { Center } \\
\text { Number }\end{array}$} & \multirow{2}{*}{$\begin{array}{l}\text { Atomic } \\
\text { Number }\end{array}$} & \multirow{2}{*}{$\begin{array}{c}\text { Atomic } \\
\text { Type }\end{array}$} & \multicolumn{3}{|c|}{ Coordinates (Angstroms) } \\
\hline & & & $\mathrm{X}$ & Y & Z \\
\hline & & & & & \\
\hline 1 & 8 & 0 & -0.568373 & -1.394586 & -0.476306 \\
\hline 2 & 6 & 0 & 0.617314 & -0.532782 & -0.833709 \\
\hline 3 & 6 & 0 & -0.332564 & -2.826043 & -0.795721 \\
\hline 4 & 56 & 0 & -2.972644 & -0.350107 & 0.194363 \\
\hline 5 & 6 & 0 & 0.228055 & 0.910840 & -0.427479 \\
\hline 6 & 6 & 0 & 1.921078 & -1.018225 & -0.235626 \\
\hline 7 & 8 & 0 & -1.002469 & 1.174114 & -0.140165 \\
\hline 8 & 1 & 0 & 0.690057 & -0.557418 & -1.931985 \\
\hline 9 & 1 & 0 & 0.505616 & -3.212690 & -0.212678 \\
\hline 10 & 1 & 0 & -0.132944 & -2.946457 & -1.866569 \\
\hline 11 & 1 & 0 & -1.245124 & -3.370440 & -0.526426 \\
\hline 12 & 7 & 0 & 1.185463 & 1.833957 & -0.450190 \\
\hline 13 & 6 & 0 & 2.931708 & -1.518914 & -1.086338 \\
\hline 14 & 6 & 0 & 2.140122 & -0.986231 & 1.163056 \\
\hline 15 & 6 & 0 & 4.149030 & -1.978846 & -0.549384 \\
\hline 16 & 6 & 0 & 0.977795 & 3.296136 & -0.176632 \\
\hline 17 & 6 & 0 & 3.350855 & -1.454269 & 1.698103 \\
\hline 18 & 1 & 0 & 2.143463 & 1.532655 & -0.625528 \\
\hline 19 & 1 & 0 & 2.776983 & -1.550178 & -2.163847 \\
\hline 20 & 1 & 0 & 1.375967 & -0.594881 & 1.833523 \\
\hline 21 & 6 & 0 & 4.358039 & -1.949848 & 0.842253 \\
\hline 22 & 6 & 0 & 1.183294 & 3.588520 & 1.324826 \\
\hline 23 & 6 & 0 & 1.930771 & 4.106392 & -1.073591 \\
\hline 24 & 1 & 0 & 4.924416 & -2.356981 & -1.208925 \\
\hline 25 & 1 & 0 & -0.061971 & 3.507405 & -0.448530 \\
\hline 26 & 1 & 0 & 3.518061 & -1.430153 & 2.771176 \\
\hline 27 & 1 & 0 & 5.296041 & -2.306216 & 1.258210 \\
\hline 28 & 1 & 0 & 0.988105 & 4.648725 & 1.520911 \\
\hline 29 & 1 & 0 & 0.500031 & 2.997525 & 1.946536 \\
\hline 30 & 1 & 0 & 2.213629 & 3.375981 & 1.635359 \\
\hline 31 & 1 & 0 & 2.982492 & 3.902336 & -0.830865 \\
\hline 32 & 1 & 0 & 1.761750 & 5.176295 & -0.913297 \\
\hline 33 & 1 & 0 & 1.767018 & 3.896185 & -2.136846 \\
\hline
\end{tabular}




\begin{abstract}
Structure: (SP, ap_2-Ba $\left.{ }^{2+}\right)$
Calculation: gas-phase b3lyp/LanL2DZ

Total energy $=-697.438199$ hartree

Cartesian coordinates:
\end{abstract}

\begin{tabular}{|c|c|c|c|c|c|}
\hline \multirow{2}{*}{$\begin{array}{l}\text { Center } \\
\text { Number }\end{array}$} & \multirow{2}{*}{$\begin{array}{l}\text { Atomic } \\
\text { Number }\end{array}$} & \multirow{2}{*}{$\begin{array}{c}\text { Atomic } \\
\text { Type }\end{array}$} & \multicolumn{3}{|c|}{ Coordinates (Angstroms) } \\
\hline & & & $\mathrm{X}$ & Y & Z \\
\hline & & & & & \\
\hline 1 & 8 & 0 & -0.555657 & -1.337658 & -0.631418 \\
\hline 2 & 6 & 0 & 0.635350 & -0.444190 & -0.861087 \\
\hline 3 & 6 & 0 & -0.324968 & -2.707382 & -1.158522 \\
\hline 4 & 56 & 0 & -2.924331 & -0.423567 & 0.281933 \\
\hline 5 & 6 & 0 & 0.233145 & 0.951799 & -0.321069 \\
\hline 6 & 6 & 0 & 1.917618 & -0.990833 & -0.267075 \\
\hline 7 & 8 & 0 & -0.990101 & 1.165269 & 0.031707 \\
\hline 8 & 1 & 0 & 0.744510 & -0.358301 & -1.953028 \\
\hline 9 & 1 & 0 & 0.515450 & -3.176492 & -0.642835 \\
\hline 10 & 1 & 0 & -0.130923 & -2.667124 & -2.236305 \\
\hline 11 & 1 & 0 & -1.237385 & -3.284743 & -0.970037 \\
\hline 12 & 7 & 0 & 1.181317 & 1.883896 & -0.279040 \\
\hline 13 & 6 & 0 & 2.957377 & -1.417461 & -1.122672 \\
\hline 14 & 6 & 0 & 2.084173 & -1.091975 & 1.135390 \\
\hline 15 & 6 & 0 & 4.150852 & -1.936532 & -0.585679 \\
\hline 16 & 6 & 0 & 0.972296 & 3.316519 & 0.121763 \\
\hline 17 & 6 & 0 & 3.270499 & -1.618805 & 1.669534 \\
\hline 18 & 1 & 0 & 2.132270 & 1.615699 & -0.530167 \\
\hline 19 & 1 & 0 & 2.844194 & -1.346526 & -2.203474 \\
\hline 20 & 1 & 0 & 1.298667 & -0.754621 & 1.810835 \\
\hline 21 & 6 & 0 & 4.306709 & -2.041039 & 0.809179 \\
\hline 22 & 6 & 0 & 2.206042 & 3.795665 & 0.907739 \\
\hline 23 & 6 & 0 & 0.693022 & 4.176549 & -1.129075 \\
\hline 24 & 1 & 0 & 4.948996 & -2.257898 & -1.248126 \\
\hline 25 & 1 & 0 & 0.089790 & 3.321293 & 0.770059 \\
\hline 26 & 1 & 0 & 3.397416 & -1.695389 & 2.745682 \\
\hline 27 & 1 & 0 & 5.226182 & -2.443259 & 1.224591 \\
\hline 28 & 1 & 0 & 2.049923 & 4.827211 & 1.240126 \\
\hline 29 & 1 & 0 & 2.388704 & 3.179304 & 1.795678 \\
\hline 30 & 1 & 0 & 3.108849 & 3.788270 & 0.281975 \\
\hline 31 & 1 & 0 & 1.553161 & 4.181309 & -1.809862 \\
\hline 32 & 1 & 0 & 0.502918 & 5.212269 & -0.826162 \\
\hline 33 & 1 & 0 & -0.186018 & 3.816706 & -1.676810 \\
\hline
\end{tabular}




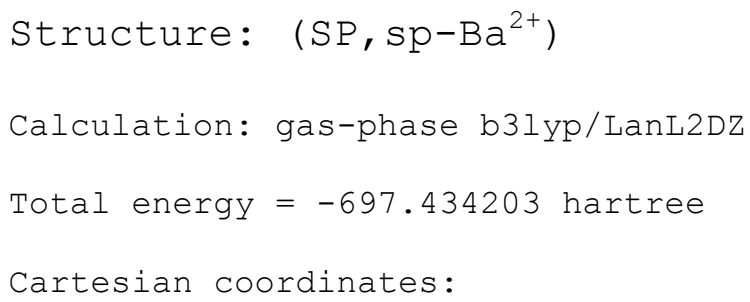

\begin{tabular}{|c|c|c|c|c|c|}
\hline \multirow{2}{*}{$\begin{array}{l}\text { Center } \\
\text { Number }\end{array}$} & \multirow{2}{*}{$\begin{array}{l}\text { Atomic } \\
\text { Number }\end{array}$} & \multirow{2}{*}{$\begin{array}{c}\text { Atomic } \\
\text { Type }\end{array}$} & \multicolumn{3}{|c|}{ Coordinates (Angstroms) } \\
\hline & & & $\mathrm{x}$ & $\mathrm{Y}$ & Z \\
\hline 1 & 8 & 0 & -0.189000 & -1.431297 & -0.493298 \\
\hline 2 & 6 & 0 & 0.809710 & -0.358354 & -0.841943 \\
\hline 3 & 6 & 0 & 0.307713 & -2.786137 & -0.847361 \\
\hline 4 & 56 & 0 & -2.729016 & -0.883086 & 0.236407 \\
\hline 5 & 6 & 0 & 0.160146 & 0.983818 & -0.409581 \\
\hline 6 & 6 & 0 & 2.186004 & -0.602399 & -0.258265 \\
\hline 7 & 8 & 0 & -1.090497 & 0.999664 & -0.096656 \\
\hline 8 & 1 & 0 & 0.876856 & -0.351499 & -1.940817 \\
\hline 9 & 1 & 0 & 1.209984 & -3.020119 & -0.278986 \\
\hline 10 & 1 & 0 & 0.514530 & -2.843035 & -1.921977 \\
\hline 11 & 1 & 0 & -0.482958 & -3.498482 & -0.585095 \\
\hline 12 & 7 & 0 & 0.942890 & 2.060668 & -0.436105 \\
\hline 13 & 6 & 0 & 3.266140 & -0.889616 & -1.122374 \\
\hline 14 & 6 & 0 & 2.404797 & -0.559383 & 1.140201 \\
\hline 15 & 6 & 0 & 4.550812 & -1.128689 & -0.598653 \\
\hline 16 & 6 & 0 & 0.540563 & 3.488877 & -0.156104 \\
\hline 17 & 6 & 0 & 3.684191 & -0.808547 & 1.661778 \\
\hline 18 & 1 & 0 & 1.928921 & 1.902874 & -0.638770 \\
\hline 19 & 1 & 0 & 3.112702 & -0.926784 & -2.199935 \\
\hline 20 & 1 & 0 & 1.586285 & -0.327656 & 1.820626 \\
\hline 21 & 6 & 0 & 4.759726 & -1.092198 & 0.792749 \\
\hline 22 & 6 & 0 & -0.523400 & 3.976582 & -1.159033 \\
\hline 23 & 6 & 0 & 0.119456 & 3.668752 & 1.316308 \\
\hline 24 & 1 & 0 & 5.378244 & -1.343384 & -1.268287 \\
\hline 25 & 1 & 0 & 1.462018 & 4.059242 & -0.324892 \\
\hline 26 & 1 & 0 & 3.851217 & -0.776613 & 2.734663 \\
\hline 27 & 1 & 0 & 5.749836 & -1.278974 & 1.198420 \\
\hline 28 & 1 & 0 & -0.701935 & 5.045447 & -0.998451 \\
\hline 29 & 1 & 0 & -0.187581 & 3.846859 & -2.194456 \\
\hline 30 & 1 & 0 & -1.472889 & 3.449116 & -1.020886 \\
\hline 31 & 1 & 0 & -0.810310 & 3.130887 & 1.530837 \\
\hline 32 & 1 & 0 & -0.051327 & 4.732917 & 1.512739 \\
\hline 33 & 1 & 0 & 0.901917 & 3.324188 & 2.002538 \\
\hline
\end{tabular}




\begin{abstract}
Structure: $\left(\mathrm{SC}, \mathrm{SC}-\mathrm{Ba}^{2+}\right)$
Calculation: gas-phase b3lyp/LanL2DZ

Total energy $=-697.382630$ hartree

Cartesian coordinates:
\end{abstract}

\begin{tabular}{|c|c|c|c|c|c|}
\hline \multirow{2}{*}{$\begin{array}{l}\text { Center } \\
\text { Number }\end{array}$} & \multirow{2}{*}{$\begin{array}{l}\text { Atomic } \\
\text { Number }\end{array}$} & \multirow{2}{*}{$\begin{array}{c}\text { Atomic } \\
\text { Type }\end{array}$} & \multicolumn{3}{|c|}{ Coordinates (Angstroms) } \\
\hline & & & $\mathrm{X}$ & Y & Z \\
\hline & & & & 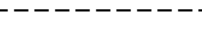 & (- \\
\hline 1 & 8 & 0 & -0.233177 & -0.861680 & 1.733000 \\
\hline 2 & 6 & 0 & -0.420109 & 0.608688 & 1.540133 \\
\hline 3 & 6 & 0 & -0.022267 & -1.266880 & 3.155510 \\
\hline 4 & 56 & 0 & 0.243717 & -1.742642 & -0.747334 \\
\hline 5 & 6 & 0 & 0.983307 & 1.046592 & 1.014836 \\
\hline 6 & 6 & 0 & -1.609416 & 0.861564 & 0.619468 \\
\hline 7 & 7 & 0 & 1.113638 & 1.090194 & -0.404911 \\
\hline 8 & 6 & 0 & -2.521847 & -0.170315 & 0.285166 \\
\hline 9 & 1 & 0 & -0.567443 & 1.075853 & 2.519379 \\
\hline 10 & 1 & 0 & -0.899845 & -0.981147 & 3.742622 \\
\hline 11 & 1 & 0 & 0.881700 & -0.786276 & 3.540161 \\
\hline 12 & 1 & 0 & 0.088531 & -2.354311 & 3.157732 \\
\hline 13 & 8 & 0 & 1.939450 & 1.109789 & 1.802667 \\
\hline 14 & 6 & 0 & -3.633148 & 0.101822 & -0.544218 \\
\hline 15 & 6 & 0 & 2.448480 & 1.486050 & -1.034221 \\
\hline 16 & 6 & 0 & -1.840791 & 2.168379 & 0.130384 \\
\hline 17 & 1 & 0 & 0.329444 & 1.591422 & -0.836411 \\
\hline 18 & 1 & 0 & -2.470739 & -1.126058 & 0.805936 \\
\hline 19 & 6 & 0 & -3.838979 & 1.399176 & -1.049635 \\
\hline 20 & 6 & 0 & 2.927278 & 2.878733 & -0.575281 \\
\hline 21 & 6 & 0 & 3.504344 & 0.388396 & -0.795754 \\
\hline 22 & 6 & 0 & -2.949212 & 2.433992 & -0.695413 \\
\hline 23 & 1 & 0 & -4.357508 & -0.680907 & -0.755549 \\
\hline 24 & 1 & 0 & 2.228717 & 1.535848 & -2.110860 \\
\hline 25 & 1 & 0 & -1.196935 & 2.993738 & 0.432710 \\
\hline 26 & 1 & 0 & -4.701411 & 1.612111 & -1.673910 \\
\hline 27 & 1 & 0 & 3.818109 & 3.161723 & -1.147251 \\
\hline 28 & 1 & 0 & 2.163410 & 3.644176 & -0.760514 \\
\hline 29 & 1 & 0 & 3.189017 & 2.886645 & 0.485923 \\
\hline 30 & 1 & 0 & 3.736571 & 0.273323 & 0.266940 \\
\hline 31 & 1 & 0 & 4.432754 & 0.652092 & -1.312581 \\
\hline 32 & 1 & 0 & 3.196321 & -0.590543 & -1.216976 \\
\hline 33 & 1 & 0 & -3.130353 & 3.446865 & -1.043436 \\
\hline
\end{tabular}




\begin{abstract}
Structure: ( $\left.\mathrm{SC},-\mathrm{ac}-\mathrm{Ba}^{2+}\right)$
Calculation: gas-phase b3lyp/LanL2DZ

Total energy $=-697.386601$ hartree

Cartesian coordinates:
\end{abstract}

\begin{tabular}{|c|c|c|c|c|c|}
\hline \multirow{2}{*}{$\begin{array}{l}\text { Center } \\
\text { Number }\end{array}$} & \multirow{2}{*}{$\begin{array}{l}\text { Atomic } \\
\text { Number }\end{array}$} & \multirow{2}{*}{$\begin{array}{c}\text { Atomic } \\
\text { Type }\end{array}$} & \multicolumn{3}{|c|}{ Coordinates (Angstroms) } \\
\hline & & & $\mathrm{X}$ & Y & Z \\
\hline & & & & & \\
\hline 1 & 8 & 0 & -0.236931 & -1.311242 & 1.500058 \\
\hline 2 & 6 & 0 & -0.655798 & 0.109749 & 1.700008 \\
\hline 3 & 6 & 0 & -0.104419 & -2.088106 & 2.769467 \\
\hline 4 & 56 & 0 & 0.604235 & -1.359763 & -1.050935 \\
\hline 5 & 6 & 0 & 0.690636 & 0.868526 & 1.524657 \\
\hline 6 & 6 & 0 & -1.767231 & 0.464171 & 0.717836 \\
\hline 7 & 7 & 0 & 0.987114 & 1.288943 & 0.187476 \\
\hline 8 & 6 & 0 & -2.465788 & -0.536318 & -0.002593 \\
\hline 9 & 1 & 0 & -0.981890 & 0.238584 & 2.737235 \\
\hline 10 & 1 & 0 & -1.075639 & -2.120973 & 3.271508 \\
\hline 11 & 1 & 0 & 0.651055 & -1.619432 & 3.406758 \\
\hline 12 & 1 & 0 & 0.203611 & -3.098214 & 2.487584 \\
\hline 13 & 8 & 0 & 1.527176 & 0.889885 & 2.438556 \\
\hline 14 & 6 & 0 & -3.517356 & -0.181592 & -0.877336 \\
\hline 15 & 6 & 0 & 2.340830 & 1.941201 & -0.045075 \\
\hline 16 & 6 & 0 & -2.149448 & 1.816803 & 0.563414 \\
\hline 17 & 1 & 0 & 0.218929 & 1.830592 & -0.223545 \\
\hline 18 & 1 & 0 & -2.311061 & -1.586395 & 0.243632 \\
\hline 19 & 6 & 0 & -3.874179 & 1.169834 & -1.043932 \\
\hline 20 & 6 & 0 & 3.416403 & 0.852919 & -0.230777 \\
\hline 21 & 6 & 0 & 2.242602 & 2.891752 & -1.248259 \\
\hline 22 & 6 & 0 & -3.197695 & 2.164946 & -0.308893 \\
\hline 23 & 1 & 0 & -4.083764 & -0.956507 & -1.387901 \\
\hline 24 & 1 & 0 & 2.588905 & 2.512357 & 0.857235 \\
\hline 25 & 1 & 0 & -1.675590 & 2.596792 & 1.158780 \\
\hline 26 & 1 & 0 & -4.693308 & 1.441520 & -1.702805 \\
\hline 27 & 1 & 0 & 4.411222 & 1.308957 & -0.265543 \\
\hline 28 & 1 & 0 & 3.429279 & 0.145096 & 0.608978 \\
\hline 29 & 1 & 0 & 3.303510 & 0.325478 & -1.199293 \\
\hline 30 & 1 & 0 & 1.980849 & 2.362150 & -2.177929 \\
\hline 31 & 1 & 0 & 3.207899 & 3.380236 & -1.415911 \\
\hline 32 & 1 & 0 & 1.505751 & 3.687078 & -1.079746 \\
\hline 33 & 1 & 0 & -3.500000 & 3.204349 & -0.399059 \\
\hline
\end{tabular}




\begin{abstract}
Structure: ( $\left.\mathrm{SC}, \mathrm{ap}-\mathrm{Ba}^{2+}\right)$
Calculation: gas-phase b3lyp/LanL2DZ

Total energy $=-697.385991$ hartree

Cartesian coordinates:
\end{abstract}

\begin{tabular}{|c|c|c|c|c|c|}
\hline \multirow{2}{*}{$\begin{array}{l}\text { Center } \\
\text { Number }\end{array}$} & \multirow{2}{*}{$\begin{array}{l}\text { Atomic } \\
\text { Number }\end{array}$} & \multirow{2}{*}{$\begin{array}{c}\text { Atomic } \\
\text { Type }\end{array}$} & \multicolumn{3}{|c|}{ Coordinates (Angstroms) } \\
\hline & & & $\mathrm{X}$ & Y & Z \\
\hline & & & & & \\
\hline 1 & 8 & 0 & -0.430337 & -1.050916 & 1.697149 \\
\hline 2 & 6 & 0 & -0.506510 & 0.442837 & 1.666533 \\
\hline 3 & 6 & 0 & -0.376231 & -1.623468 & 3.076645 \\
\hline 4 & 56 & 0 & 0.191497 & -1.684278 & -0.839042 \\
\hline 5 & 6 & 0 & 0.971213 & 0.838292 & 1.384574 \\
\hline 6 & 6 & 0 & -1.540257 & 0.882297 & 0.634139 \\
\hline 7 & 7 & 0 & 1.319590 & 0.914365 & -0.003245 \\
\hline 8 & 6 & 0 & -2.506029 & -0.022068 & 0.124860 \\
\hline 9 & 1 & 0 & -0.766407 & 0.802685 & 2.667403 \\
\hline 10 & 1 & 0 & -1.292019 & -1.354686 & 3.611018 \\
\hline 11 & 1 & 0 & 0.507126 & -1.241343 & 3.596472 \\
\hline 12 & 1 & 0 & -0.316004 & -2.708760 & 2.962833 \\
\hline 13 & 8 & 0 & 1.807110 & 0.842970 & 2.298523 \\
\hline 14 & 6 & 0 & -3.485312 & 0.421402 & -0.792193 \\
\hline 15 & 6 & 0 & 2.763970 & 1.238949 & -0.348838 \\
\hline 16 & 6 & 0 & -1.585022 & 2.235696 & 0.226349 \\
\hline 17 & 1 & 0 & 0.671480 & 1.520097 & -0.521934 \\
\hline 18 & 1 & 0 & -2.600746 & -1.014202 & 0.565395 \\
\hline 19 & 6 & 0 & -3.505515 & 1.765232 & -1.211707 \\
\hline 20 & 6 & 0 & 3.096968 & 0.625690 & -1.721834 \\
\hline 21 & 6 & 0 & 3.004744 & 2.760772 & -0.311883 \\
\hline 22 & 6 & 0 & -2.562591 & 2.672772 & -0.687382 \\
\hline 23 & 1 & 0 & -4.254434 & -0.264374 & -1.138844 \\
\hline 24 & 1 & 0 & 3.372627 & 0.767957 & 0.429973 \\
\hline 25 & 1 & 0 & -0.900384 & 2.965754 & 0.656902 \\
\hline 26 & 1 & 0 & -4.268620 & 2.110614 & -1.902569 \\
\hline 27 & 1 & 0 & 4.119783 & 0.885521 & -2.013475 \\
\hline 28 & 1 & 0 & 3.067461 & -0.480734 & -1.706986 \\
\hline 29 & 1 & 0 & 2.436946 & 1.007576 & -2.515448 \\
\hline 30 & 1 & 0 & 2.412779 & 3.283022 & -1.076892 \\
\hline 31 & 1 & 0 & 4.060339 & 2.979044 & -0.506148 \\
\hline 32 & 1 & 0 & 2.761103 & 3.173761 & 0.672549 \\
\hline 33 & 1 & 0 & -2.602753 & 3.720218 & -0.972148 \\
\hline
\end{tabular}

